\title{
JORDAN RINGS WITH INVOLUTION
}

\author{
BY \\ NG SEONG-NAM $\left(^{1}\right)$
}

\begin{abstract}
Let $J$ be a Jordan ring with involution ${ }^{*}$ in which $2 x=0$ implies $x=0$ and in which $2 J=J$. Let the set $S$ of symmetric elements of $J$ be periodic and let $N$ be the Jacobson radical of $J$. Then $N^{2}=0$ and $J / N$ is a subdirect sum of ${ }^{*}$-simple Jordan rings of the following types (1) a periodic field, (2) a direct sum of two simple periodic Jordan rings with exchange involution, (3) a $3 \times 3$ or $4 \times 4$ Jordan matrix algebra over a periodic field, (4) a Jordan algebra of a nondegenerate symmetric bilinear form on a vector space over a periodic field.
\end{abstract}

1. Introduction. Recently, Osborn and S. Montgomery both independently determined the structure of an associative ring with involution in which each symmetric element is periodic. They proved:

TheOREM 1.1 (OSBORn [3, p. 363] OR MONTGOMERy [1]). Let $R$ be an associative ring with involution in which $2 a=0$ implies $a=0$ and in which $2 R=R$. Let the set $H$ of symmetric elements of $R$ be periodic and let $N$ be the Jacobson radical of $R$. Then $N^{2}=0$ and $R / N$ is a subdirect sum of rings with involution each of which is one of the following types:

(i) a periodic field,

(ii) a direct sum of two periodic fields interchanged by the involution,

(iii) a $2 \times 2$ matrix algebra over a periodic field.

The main object of this paper is to generalize the above result to a Jordan ring with involution. For convenience we adopt the following convention:

Convention. Throughout this paper, the letter $J$ will represent a Jordan ring with involution ${ }^{*}$ in which $2 a=0$ implies $a=0$ and in which $2 J=J$ and the letters $S$ and $K$ will represent the set of symmetric elements and the set of skew elements respectively.

Received by the editors September 29, 1972.

AMS (MOS) subject classifications (1970). Primary 17C10, 17C20, $17 \mathrm{C} 30$.

(1) This paper is part of the author's Ph.D. dissertation prepared at the University of Iowa under the guidance of Professor Frank Kosier. The author wishes to express his sincere thanks to Professor Frank Kosier for suggesting the problem and for his guidance. 
We shall show first that if $J$ is ${ }^{*}$-prime and $S$ is periodic then $S$ cannot contain more than four orthogonal idempotents. We classify $J$ according to the number of orthogonal idempotents that $S$ contains. Then we prove our main theorem as stated in the abstract.

2. Preliminary results. By a Jordan ring $R$, we shall mean a nonassociative ring satisfying $x \cdot y=y \cdot x,\left(x^{2} \cdot y\right) \cdot x=x \cdot\left(y^{2} \cdot x\right), 2 x=0$ implies $x=0$. If $R$ is also a vector space over a field $F$ of characteristic not two such that $(f x) \cdot y=(x f) \cdot y=x \cdot(f y)$ for all $x, y$ in $R$ and $f$ in $F$, then $R$ is called a Jordan algebra over $F$. We define $U_{a, b}=(a \cdot x) \cdot b+(b \cdot x) \cdot$ $a-(a \cdot b) \cdot x$ and abbreviate $U_{a, a}=U_{a}$ in any Jordan ring $R$. The following identities are important (see Jacobson [1, p. 34]).

$$
\begin{gathered}
(a \cdot(b \cdot c)) \cdot d+(a \cdot(b \cdot d)) \cdot c+(a \cdot(c \cdot d)) \cdot b \\
=((a \cdot b) \cdot c) \cdot d+((a \cdot d) \cdot c) \cdot b+a \cdot((b \cdot d) \cdot c) \\
=(a \cdot b) \cdot(c \cdot d)+(a \cdot d) \cdot(b \cdot c)+(a \cdot c) \cdot(b \cdot d) \quad \text { (Jordan identities), } \\
\quad U_{a} U_{b}=U_{b} U_{a} U_{b} .
\end{gathered}
$$

An element $a$ of a Jordan ring $R$ is said to be invertible if $U_{a}$ is bijective and $a$ is an absolute zero divisor if $U_{a}=0$. A Jordan division ring is a Jordan ring in which each nonzero element is invertible. An involtation ${ }^{*}$ on a nonassociative ring $D$ is a map of $D$ to $D$ which satisfies $(a+b)^{*}=a^{*}+b^{*},(a b)^{*}=$ $b^{*} a^{*},\left(a^{*}\right)^{*}=a$ for all $a$ and $b$ in $D$. Let $D$ be an arbitrary nonassociative algebra of characteristic not two with identity 1 and involution ${ }^{*}$, and let $D_{n}$ be the algebra of $n \times n$ matrices with entries in $D$. Define $J_{a} x=a^{-1}\left(x^{*}\right)^{t} a$ where $a=\operatorname{diag}\left\{a_{1}, \cdots, a_{n}\right\}$ and the $a_{i}$ satisfy $a_{i}^{*}=a_{i}, a_{i} \in N(D)$, the nucleus of $D$ and $a_{i}^{-1}$ exists in $N(D)$. Let $H\left(D_{n}, J_{a}\right)$ be the set of symmetric elements relative to the involution $J_{a}$. Then $H\left(D_{n}, J_{a}\right)$ will be called the $n \times n$ Jordan matrix algebra. We will have occasions to use the following theorems.

THEOREM 2.1 (SHIRSHOV-COHN) (JACOBSON [1, p. 77]). Let $R$ be a Jordan algebra with 1 which is generated by two elements. Then $R$ is isomorphic to a Jordan algebra $S\left(C,{ }^{*}\right)$ where $C$ is an associative algebra with 1 and an involution ${ }^{*}, S\left(C,{ }^{*}\right)$ is the symmetric part of the involution *.

THEOREM 2.2 (JACOBSON [1, p. 61]). Let $D$ be an algebra over a field of characteristic not two with an identity element and an involution *, and let $J_{a}$ be a canonical involution in $D_{n}$. Then $H\left(D_{n}, J_{a}\right)$ for $n \geqslant 3$ is Jordan if and only if either $D$ is associative or $n=3$ and $D$ is alternative with symmetric elements in the nucleus. 
ThEOREM 2.3 (JACOBSON [1, p. 129]). Let $R=H\left(D_{n}, J_{a}\right), n \geqslant 3$, be a Jordan matrix algebra. Then the mapping $I \rightarrow R \cap I_{n}$ is a lattice isomorphism of the lattice of ${ }^{*}$ ideals of $D$ onto the lattice of ideals of $R$. Also $I^{2}=0$ if and only if $\left(R \cap I_{n}\right) \cdot\left(R \cap I_{n}\right)=0$.

THEOREM 2.4 (J ACOBSON [1, p. 119]). Let $R=R_{1}(e)+R_{1 / 2}(e)+R_{0}(e)$ be the Peirce decomposition of a Jordan ring $R$ relative to the idempotent $e$. Then

$$
\begin{aligned}
& R_{i}^{2} \subseteq R_{i} \quad(i=0,1), \quad R_{1 / 2} \cdot\left(R_{0}+R_{1}\right) \subseteq R_{1 / 2}, \\
& R_{1 / 2}^{2} \subseteq R_{0}+R_{1}, \\
& R_{0} \cdot R_{1}=0 . \\
& x_{1 / 2} \cdot\left(a_{i} \cdot b_{i}\right)=\left(x_{1 / 2} \cdot a_{i}\right) \cdot b_{i}+\left(x_{1 / 2} \cdot b_{i}\right) \cdot a_{i} \\
& \text { if } x_{1 / 2} \in R_{1 / 2}, a_{i}, b_{i} \in R_{i} \text { for } i=0,1 . \\
& \left(x_{1 / 2} \cdot a_{0}\right) \cdot a_{1}=\left(x_{1 / 2} \cdot a_{1}\right) \cdot a_{0} \\
& \text { if } a_{i} \in R_{i} \text { for } i=0,1 \text { and } x_{1 / 2} \in R_{1 / 2} \cdot \\
& \left(x_{1 / 2} \cdot y_{1 / 2}\right) \cdot z_{i}=\left(\left(x_{1 / 2} \cdot z_{i}\right) \cdot y_{1 / 2}\right) \cdot e_{i}+\left(\left(y_{1 / 2} \cdot z_{i}\right) \cdot x_{1 / 2}\right) \cdot e_{i} \\
& \text { if } z_{i} \in R_{i} \text { for } i=0,1 \text { and } x_{1 / 2}, y_{1 / 2} \in R_{1 / 2} .
\end{aligned}
$$

An element $z$ in a Jordan algebra $R$ over a field $F$ is quasi-invertible with quasi-inverse $w$ in $R$ if $1-z$ is invertible with inverse $1-w$ in the Jordan algebra $R^{1}=F 1+R$. An ideal is quasi-invertible if all its elements are quasi-invertible. For any Jordan algebra there is a unique maximal quasi-invertible ideal called the Jacobson radical containing all quasi-invertible ideals (see McCrimmon [2]). We call a Jordan algebra semisimple if its Jacobson radical is zero.

ThEOREM 2.5 (MCCRIMMON [3]). For any idempotent $e$ in a Jordan algebra $R$, the Jacobson radical of Peirce subalgebra $R_{1}(e)=R \cup e$ is $\operatorname{rad}(R \cup e)=(R \cup e) \cap \operatorname{rad} R=(\operatorname{rad} R) \cup e$.

THEOREM 2.6 (TSAI [1]). The intersection of all prime ideals of a Jordan ring $R$ is defined as the prime radical of $R$. If $R$ contains a maximal nilpotent ideal $N$, then the prime radical of $R$ is equal to $N$.

An element $a$ of a Jordan ring is said to be periodic if $a^{n(a)}=a$ for some positive integer $n(a)$ greater than one. A periodic Jordan ring is a Jordan ring in which each element is periodic. 
ThEOREM 2.7 (OSBORN [3, p. 342]). A periodic Jordan division ring is a periodic field.

ThEOREM 2.8 (OSBORN [3, p. 343]). Let $\mu$ and $\nu$ be elements of $a$ periodic field $F$ of characteristic $p \neq 2$. Then there exists elements $a, b, c \in$ $F$ not all zero such that $a^{2}+b^{2} \mu+c^{2} \nu=0$.

THEOREM 2.9 (OSBORN [3, p. 351]). Let $R$ be a periodic Jordan ring and let $e, f, g$ be pairwise orthogonal idempotents of $R$ such that $e$ and $f$ are connected. Then $R_{1 / 2}(g) \cap R_{1 / 2}(e)=0$.

THEOREM 2.10 (OSBORN [3, p. 345]). Let $R$ be a simple periodic Jordan ring. Then $R$ is either a periodic field or $R$ is isomorphic to the Jordan algebra

$$
B=\left\{\left[\begin{array}{ll}
a & b \mu \\
b & c
\end{array}\right] \begin{array}{l}
a, b, c \text { are elements of a perodic } \\
\text { field } F \text { and }-\mu \text { is a nonsquare in } F
\end{array}\right\} \text {. }
$$

THEOREM 2.11 (OSBORN [2]). If all elements of a Jordan ring are either invertible or nilpotent, then the set of all nilpotent elements forms an ideal.

THEOREM 2.12 (JACOBSON $[1, \mathrm{p} .170])$. Let $R$ be a *-simple alternative algebra with involution and identity element such that every nonzero symmetric element of $R$ is invertible and in the nucleus and let $T$ be the subset of $R$ of symmetric elements of the center. Then $R$ is one of the following:

(1) a direct sum of two associative division algebras interchanged by the involution,

(2) an associative division algebra with involution,

(3) a $2 \times 2$ matrix algebra over $T$ with standard involution, i.e.

$$
\left(\begin{array}{ll}
a & b \\
c & d
\end{array}\right)^{*}=\left(\begin{array}{cc}
d & -b \\
-c & a
\end{array}\right)
$$

(4) a Cayley-Dickson algebra over $T$ with standard involution.

Conversely, any algebra in the classes (1) to (4) satisfy the given conditions.

Two orthogonal idempotents $e_{1}$ and $e_{2}$ in a Jordan ring $R$ are said to be connected if there exists an element in $R_{12}=R_{1 / 2}\left(e_{1}\right) \cap R_{1 / 2}\left(e_{2}\right)$ invertible in $R U_{e_{1}+e_{2}}=R_{11}+R_{12}+R_{22}$. If $1=e_{1}+e_{2}, R_{11}$ and $R_{22}$ are Jordan division rings, then $R$ is said to be of capacity two.

THEOREM 2.13 (OSBORN [1]). Let $R$ be a simple Jordan algebra of capacity 2. Then either $R$ is isomorphic to the Jordan algebra of a nondegenerate symmetric bilinear form $f$ on a vector space over an extension field $T$ of the base field $F$, such that $f(x, x)=1$ for some $x$, or $R$ is isomorphic to a Jordan matrix algebra $H\left(D_{2}, J_{a}\right)$ where $(D, j)$ is either of the form $B \oplus B$, 
$B$ an associative division algebra which is not commutative, $j$, the exchange involution or $D$ is a division algebra which is not commutative and is not a quaternion algebra over its center with $j$ the standard involution.

Theorem 2.14 (CoORdinatization THEOREM) (J ACOBSON [1, p. 133]). Let $R=\Sigma_{i \leqslant j}^{n} R_{i j}$ be the Peirce decomposition with respect to the $e_{i}$ and $n \geqslant 3$. Assume that for $j=2,3, \cdots, n$ there exists an element $u_{1 j} \in R_{1 j}$ which is invertible in $R_{11}+R_{j j}+R_{1 j}$. Then there exists a Jordan matrix algebra $H\left(D_{n}, J_{a}\right)$ and an isomorphism $f$ of $R$ onto $H\left(D_{n}, J_{a}\right)$ such that $f\left(e_{i}\right)=1 / 2[i i], f\left(u_{1 j}\right)=[1 j]$.

3. Classification of semisimple Jordan rings when $S$ is a periodic Jordan division ring. We shall show in this section that the set of elements of the type $a=s+k$ where $s^{*}=s, k^{*}=-k, s \neq 0$ and $k^{2} \neq 0$ determines the structure of $J$ in case $J$ is semisimple and $S$ is a periodic Jordan division ring. If they are all invertible, then $J$ is a periodic field. If not, then $J$ is a direct sum of two isomorphic periodic fields or a Jordan algebra of a nondegenerate symmetric bilinear form over a periodic field. We first prove the following theorem:

THEOREM 3.1. If $J$ contains no nonzero ideal $I$ such that $I^{2}=0$ and no nonzero $s \in S, s^{2}=0$, then the identity of $S$ is the identity of $J$.

Proof. Let $e$ be the identity of $S$ and let $J=J_{1}+J_{1 / 2}+J_{0}$ be the Peirce decomposition of $J$ with respect to $e$. Then $S \subseteq J_{1}$, since $e \cdot s=s$ for all $s \in S$. If we write $x_{0} \in J_{0}$ as a sum of symmetric and skew elements $s+k$, then $0=x_{0} \cdot e=s \cdot e+k \cdot e=s+k \cdot e$ and $-s=k \cdot e$ is both skew and symmetric. Thus $s=0$. By the same argument, we can show all elements in $J_{1 / 2}$ are skew. But

$$
J_{0}^{2} \subseteq S \cap J_{0}=0, \quad J_{1 / 2} \cdot J_{0} \subseteq J_{1 / 2} \cap S=0, \quad J_{1} \cdot J_{0}=0,
$$

and so $J_{0}$ is an ideal whose square is equal to zero. Thus $J_{0}=0$ and $J=$ $J_{1}+J_{1 / 2}$.

Let $x \in J_{1 / 2}$. Then $x$ is skew and $x^{2} \in S \subseteq J_{1}$. Since $x^{3}=\left(x^{2} \cdot e\right) \cdot x$ $=x^{2} \cdot(e \cdot x)=2^{-1} x^{3}$, we have $x^{3}=0=x^{4}$. But $S$ has no trivial elements, so $x^{2}=0$. This implies $J_{1 / 2}^{2}=0$ and $J_{1 / 2}$ is a trivial ideal of $J$. Therefore $J_{1 / 2}=0$ and $e$ is the identity of $J$.

LEMMA 3.2. Let $J$ be generated by two elements $s$ and $k$ where $s^{*}=$ $s \neq 0, k^{*}=-k$ and $k^{2} \neq 0$ and let $S$ be a periodic Jordan division ring. Then

(i) $J$ is isomorphic to the set of symmetric elements of an associative ring $C$ with involution $j$, which is generated by two elements $s$ and $k$. 
If we use juxtaposition for multiplication in $C$ and a dot for multiplication in $J$, then the following are true.

(ii) $s h=h s$ in the associative ring $C$ where $s$ and $h$ are arbitrary elements of $S$.

(iii) $S=S(+)+S(-)$ where $S(+)=\{h \in S \mid h k=k h\}=\{h \in S \mid(h \cdot k) \cdot$ $\left.k=h \cdot k^{2}\right\}$ and $S(-)=\{h \in S \mid-h k=k h\}=\{h \in S \mid h \cdot k=0\}$ such that $S(+) \cdot S(+) \subseteq S(+), S(-) \cdot S(-) \subseteq S(+), S(+) \cdot S(-) \subseteq S(-)$.

(iv) $J$ is a finite dimensional Jordan algebra over $S(+)$.

Proof. (i) This follows easily from Theorem 2.1.

(ii) $S$ forms a periodic field by Theorem 2.7. Let $\langle s, h\rangle$ be the Jordan subring of $S$ generated by $s$ and $h$. The $\langle s, h\rangle$ is a finite field. Thus $s=$ $s_{1}^{i}, h=s_{1}^{m}$ for some $s_{1} \in\langle s, h\rangle$ and positive integers $i$ and $m$ and so $s h=h s=s_{1}^{i+m}$ in the associative ring $C$.

(iii) From part (ii), it follows that any element $w \in C$ is of the form $w=s_{0}+\sum_{i=1}^{n} s_{i} k h_{i}$ where $\left\{s_{0}, s_{i}, h_{i}\right\}_{i=1}^{n} \subseteq S$. But $0 \neq k^{2} \in S$ and $S$ is a periodic field, so $k^{2 p+2}=1$ for some $p \geqslant 0$. Thus

$$
w=s_{0}+\sum_{i=1}^{n} s_{i} k h_{i} k k^{-1}=s_{0}+\sum_{i=1}^{n} s_{i}\left(k h_{i} k\right) k^{2 p+1}=s_{0}+\sum_{i=1}^{n} s_{i}\left(k h_{i} k\right) k^{2 p} k .
$$

This means $C=S+S k$ is a two dimensional left vector space over $S$. But for any $q \in S, j(q k)=j(k) j(q)=k q=k q k k^{-1}=(k q k) k^{2 p+1}=(k q k)\left(k^{2 p}\right) k \in$ $S k$. Thus any $q k \in S k$ can be written as $q k=(q(+)+q(-)) k$ satisfying $j(q(+) k=$ $q(+) k=k q(+)$ and $j(q(-) k)=-q(-) k=k q(-), q=q(+)+q(-)$, since $k$ is invertible. But $q(+)$ commutes with $s$ by part (1) and $C$ is generated by $s$ and $k$, so $q(+) \in$ the center of $C$. Hence we have proved that any $q \in S$ can be written as $q=q(+)+q(-)$ such that $q(+) k=k q(+)$ and $q(-) k=-k q(-)$. If $h_{1}, h_{2} \in S(+)$, then $\left(h_{1} h_{2}\right) k=h_{1}\left(h_{2} k\right)=h_{1}\left(k h_{2}\right)=k\left(h_{1} h_{2}\right)$ and so $S(+) S(+) \subseteq S(+)$. The same arguments show $S(+) S(-) \subseteq S(-), S(-) S(+) \subseteq$ $S(-), S(-) S(-) \subseteq S(+)$. Hence we have $S(+) \cdot S(+) \subseteq S(+), S(+) \cdot S(-) \subseteq$ $S(-), S(-) \cdot S(-) \subseteq S(+)$.

(iv) Since $C=S(+)+S(+) h+S(+) k+S(+)(h k)$ where $h \in S(-)$ is finite dimensional over $S(+)$ and $J$ is a subspace of $C$, so $J$ is a finite dimensional Jordan algebra over $S(+)$.

Theorem 3.3. Let $J$ be a Jordan division ring and $S$ be periodic. Then $J$ is a periodic field.

Proof. Let $a=s+k$ where $0 \neq s \in S$ and $0 \neq k \in K$ and let $A$ be the Jordan subring generated by $s$ and $k$. Let $H$ denote the set of * symmetric elements in $A$. Then by Lemma 3.2, $A$ is isomorphic to the sym- 
metric part of an associative ring $C$ with involution $j$ generated by $s$ and $k$, such that $H=H(+)+H(-)$ and $A$ is a finite dimensional algebra over $H(+)$. Suppose $a$ satisfies an equation $x^{n}+b_{1} \cdot x^{n-1}+\cdots+b_{n}=0$ with coefficients in $H(+)$. Let $B=\left\langle b_{1}, \cdots, b_{n}\right\rangle$ be the finite subfield of $H(+)$ generated by $b_{1}, b_{2}, \cdots, b_{n}$. Then $B[a]$ is a finite associative division ring and $a$ is periodic. Therefore $J$ is a periodic field by applying Osborn's Theorem 2.7.

Lemma 3.4. Let $F$ be a finite field and $G, H$ be two subfields. If $[F: G]=[F: H]=2$, then $G=H$.

Proof. Let $p$ be the characteristic of $F$ and $|G|=p^{n}$. If $G \neq H$, then $x^{p^{n}}-x \in F[x]$ has more than $p^{n}$ roots in $F$. This cannot happen.

Lemma 3.5. Let $F$ be a periodic field and $G, H$ be two subfields. If $[F: G]=[F: H]=2$, then $G=H$.

Proof. Let $p$ be the characteristic of $F$. Suppose $G \neq H$ and $g \in G$, $g \notin H$. Let $i$ and $j$ be the involutions of $F$ over $H$ and $F$ over $G$ respectively. The elements $g, i(g), j(i(g)), i(j(i(g))), \cdots$, satisfy an algebraic equation of $g$ over $I /[p]$ and so they are finite in number. Let $W_{1}$ be the subfield generated by these elements. Then $W_{1}$ is a finite subfield which is invariant under $i$ and $j$. Similarly if $h \in H, h \notin G$, we can construct another invariant subfield $W_{2}, h \in W_{2}$. Then the finite subfield $W$ generated by $W_{1}$ and $W_{2}$ has two involutions $i$ and $j$ and so by Lemma 3.4, $G \cap W=H \cap W$. This contradicts $g \in G \cap W$ but $g \notin H \cap W$.

Lemma 3.6. Any two quadratic extensions of a periodic field are isomorphic.

Proof. Let $F$ be a periodic field, $F[x]$ and $F[y]$ by any two quadratic extensions of $F$ such that $x^{2}-a x+b=0$ and $y^{2}-c y+d=0$ where $a, b, c, d \in F$. Let $B$ denote the subfield of $F$ generated by $a, b, c$, d. Then $B$ is a finite field and so $B[x]$ is isomorphic to $B[y]$. We can find $w$ in $B[x]$ such that $w^{2}-c w+d=0$. Hence $F[x]$ and $F[y]$ are the splitting fields of the same polynomial. Therefore they are isomorphic.

Lemma 3.7. Let $J$ be a Jordan ring with involution and $S$ be a periodic division ring. Let $0 \neq s \in S, k \in K$ and $k^{2}=0$. Then $(s+k)^{m}=1$ for some positive integer $\mathrm{m}$.

PROOF. By Shirshov's Theorem 2.1, the Jordan subring $\langle s, k\rangle$ generated by $s$ and $k$ is a subring of $C^{+}$where $C$ is an associative ring. Since $k(k s k)=k^{2} s k=0$ in $C$ and $k s k$ is invertible or zero, we have $k s k=0$. 
This implies

$$
\begin{aligned}
& a^{2}=(s+k)^{2}=s^{2}+k s+s k \text { in } C, \\
& a^{3}=s^{3}+k s^{2}+s k s+s^{2} k, \\
& \cdots \\
& a^{n}=s^{n}+k s^{n-1}+s k s^{n-1}+s^{2} k s^{n-1}+\cdots+s^{n-1} k \text { in } C .
\end{aligned}
$$

Let $n$ be any positive integer such that $s^{n}=1$. Then,

$$
\begin{aligned}
a^{n} & =1+k s^{n-1}+s k s^{n-2}+\cdots+s^{n-1} k, \\
a^{2 n} & =1+2\left(k s^{n-1}+s k s^{n-2}+\cdots+s^{n-1} k\right), \\
\cdots & \\
a^{p n} & =1+p\left(k s^{n-1}+\cdots+s^{n-1} k\right) \\
& =1 \text { if } p \text { is the characteristic of } C .
\end{aligned}
$$

LemMA 3.8. Let $J$ be a Jordan ring with involution and $S$ be a periodic Jordan division ring. Then for any $k \in K, k^{2} \neq 0$, we can decompose $S=$ $S(+)+S(-)$ where $S(+)=\{s \in S \mid(s, k, k)=0\}$ and $S(-)=\{s \in S \mid s \cdot k=0\}$ such that

(1) $S(+)^{2} \subseteq S(+), S(+) \cdot S(-) \subseteq S(-), S(-)^{2} \subseteq S(+)$,

(2) $(s \cdot h) \cdot k=s \cdot(h \cdot k)$ for $s, h \in S(+)$.

Proof. We have shown in Lemma 3.2 that any $s \in S$ can be written as $s=s_{+}+s_{-}$such that $\left(s_{+}, k, k\right)=0$ and $s_{-} \cdot k=0$ relative to an invertible skew element $k$. Thus $S=S(+)+S(-)$. Let $s, h \in S(+), s_{-} \in S(-)$. We now show $s \cdot h \in S(+), s_{-}^{2} \in S(+), s \cdot s_{-} \in S(-)$.

Let $W$ be the Jordan subring generated by $s, h$ and $s_{-}$. Then $W$ is a finite field and so $s=w^{m}, h=w^{n}, s_{-}=w^{l}$ for some positive integers $m$, $n, l$ and $w \in W$. Consider the Jordan subring $\langle w, k\rangle$ generated by $w$ and $k$. Then $H=S \cap\langle w, k\rangle$ can be decomposed as $H=H(+)+H(-)$ where $H(+)=$ $\{t \in H \mid(t, k, k)=0\}, H(-)=\{t \mid t \cdot k=0\}$ by Lemma 3.2. But $s, h \in$ $H(+), s_{-} \in H(-)$. Hence by Lemma 3.2, $s \cdot h \in H(+) \subseteq S(+), s \cdot s_{-} \in$ $H(-) \subseteq S(-), s_{-}^{2} \in H(+)$, and also $(s \cdot h) \cdot k=s \cdot(h \cdot k)$.

LEMMA 3.9. Let $J$ be a simple Jordan ring with involution and $S$ be a periodic Jordan division ring. Let $S=S(+)+S(-)$ be a decomposition relative to an invertible skew element $k_{1}$. If $k_{1}^{2}=s^{2}$ for some $s \in S(+)$, then $J$ is a Jordan algebra of a nondegenerate symmetric bilinear form over a subfield of $S$.

Proof. Let $e_{1}=1 / 2\left(1-k_{1} \cdot s^{-1}\right)$ and $e_{2}=1 / 2\left(1+k_{1} \cdot s^{-1}\right)$. Then $e_{1}$ and $e_{2}$ are two orthogonal idempotents such that $1=e_{1}+e_{2}$ and $e_{1}^{*}=$ 
$e_{2}$. Let $J=J_{1}+J_{1 / 2}+J_{0}$ be the Peirce decomposition of $J$ with respect to $e_{1}$. We claim $a_{1} \cdot a_{1 / 2}=a_{1}^{*} \cdot a_{1 / 2}$ for all $a_{1} \in J_{1}$ and $a_{1 / 2} \in J_{1 / 2}$. If $S \cap J_{1 / 2}=0$, then all elements of $J_{1 / 2}$ are skew and so $\left(a_{1}-a_{1}^{*}\right) \cdot a_{1 / 2} \in S \cap J_{1 / 2}=0$. Hence assume $S \cap J_{1 / 2} \neq 0$. We first prove it is true for any $h \in S \cap J_{1 / 2} \neq 0$.

From $\left(a_{1}+a_{1}^{*}\right) \cdot\left[\left(a_{1}+a_{1}^{*}\right) \cdot h\right]=\left(a_{1}+a_{1}^{*}\right)^{2} \cdot h$, it follows that

$$
\begin{aligned}
a_{1} \cdot\left(a_{1} \cdot h\right)+a_{1} \cdot\left(a_{1}^{*} \cdot h\right) & +a_{1}^{*} \cdot\left(a_{1} \cdot h\right)+a_{1}^{*} \cdot\left(a_{1}^{*} \cdot h\right) \\
& =a_{1}^{2} \cdot h+a_{1}^{* 2} \cdot h=2\left(a_{1} \cdot h\right) \cdot a_{1}+2\left(a_{1}^{*} \cdot h\right) \cdot a_{1}^{*}
\end{aligned}
$$

and so $\left(a_{1}-a_{1}^{*}\right) \cdot\left[\left(a_{1}-a_{1}^{*}\right) \cdot h\right]=0$. If we write $h=h(+)+h(-)$ relative to $0=a_{1}-a_{1}^{*}$, then $\left(a_{1}-a_{1}^{*}\right)^{2} \cdot h(+)=0$. This gives us $h=h(-)$ and $h \cdot\left(a_{1}-a_{1}^{*}\right)=0$. Let $k$ be any skew element in $J_{1 / 2}$. Since $\left(a_{1}-a_{1}^{*}\right) \cdot k$ is symmetric, $\left(a_{1}-a_{1}^{*}\right) \cdot\left[\left(a_{1}-a_{1}^{*}\right) \cdot k\right]=0$. But

$$
\begin{aligned}
2\left[\left(a_{1}-a_{1}^{*}\right) \cdot k\right] \cdot\left[\left(a_{1}-a_{1}^{*}\right) \cdot k\right] & \\
= & -\left(a_{1}-a_{1}^{*}\right)^{2} \cdot k^{2}+2\left[\left(a_{1}-a_{1}^{*}\right) \cdot\left(k \cdot\left(a_{1}-a_{1}^{*}\right)\right)\right] \\
& \cdot k+\left[\left(a_{1}-a_{1}^{*}\right) \cdot k^{2}\right] \cdot\left(a_{1}-a_{1}^{*}\right) \text { by the Jordan identity, } \\
= & \left(a_{1}-a_{1}^{*}\right)^{2} \cdot k^{2}+k^{2} \cdot\left(a_{1}-a_{1}^{*}\right)^{2}=0 .
\end{aligned}
$$

This implies $\left[\left(a_{1}-a_{1}^{*}\right) \cdot k\right]^{2}=0$ and so $\left(a_{1}-a_{1}^{*}\right) \cdot k=0$.

$*$ Let $h \in S \cap J_{1 / 2}$ and $k \in K \cap J_{1 / 2}$. Since $h \cdot k \in J_{1}+J_{0}$ and is skew, we can assume $h \cdot k=a_{1}-a_{1}^{*}$ where $a_{1}=[h \cdot k]_{1}$. Then $(h \cdot k) \cdot k=0$ and so $2[h \cdot k] \cdot[h \cdot k]=-h^{2} \cdot k^{2}+2[h \cdot(k \cdot h)] \cdot k+\left(h \cdot k^{2}\right) \cdot h=0$. Therefore $(h \cdot k)^{2}=\left(a_{1}-a_{1}^{*}\right)^{2}=\left(a_{1}+a_{1}^{*}\right)^{2}=0$ which implies $a_{1}=a_{1}^{*}=0$ and $h \cdot k=0$.

We next prove $T=\left\{a_{1}+a_{1}^{*} \mid a_{1} \in J_{1}\right\}$ is contained in the center of $J$. The nontrivial verifications required to establish this are (1) $\left(b_{1}, x_{1 / 2}, a_{1}+a_{1}^{*}\right)=$ 0 , (2) $\left(b_{1 / 2}, x_{1}, a_{1}+a_{1}^{*}\right)=0$, (3) $\left(b_{1 / 2}, x_{1 / 2}, a_{1}+a_{1}^{*}\right)=0$,

$$
\begin{aligned}
& \left.\left(b_{1}, x_{1 / 2}, a_{1}+a_{1}^{*}\right)=\left(b_{1}, x_{1 / 2}, a_{1}\right) \quad \text { (Theorem } 2.4\right) \\
& =\left(b_{1} \cdot x_{1 / 2}\right) \cdot a_{1}-b_{1} \cdot\left(x_{1 / 2} \cdot a_{1}\right)=\left(b_{1} \cdot x_{1 / 2}\right) \cdot a_{1}^{*}-b_{1} \cdot\left(x_{1 / 2} \cdot a_{1}\right) \\
& =\left(a_{1}^{*} \cdot x_{1 / 2}\right) \cdot b_{1}-b_{1} \cdot\left(x_{1 / 2} \cdot a_{1}\right)=\left(a_{1} \cdot x_{1 / 2}\right) \cdot b_{1}-b_{1} \cdot\left(x_{1 / 2} \cdot a_{1}\right)=0, \\
& \left(b_{1 / 2}, x_{1}, a_{1}+a_{1}^{*}\right)=\left(b_{1 / 2} \cdot x_{1}\right) \cdot\left(a_{1}+a_{1}^{*}\right)-b_{1 / 2} \cdot\left(x_{1} \cdot\left(a_{1}+a_{1}^{*}\right)\right) .
\end{aligned}
$$

But $y_{1} \cdot y_{1 / 2}=y_{1}^{*} \cdot y_{1 / 2}$ for all $y_{1} \in J_{1}$ and $y_{1 / 2} \in J_{1 / 2}$. Thus

$$
\begin{aligned}
& \left(b_{1 / 2}, x_{1}, a_{1}+a_{1}^{*}\right)=\left(b_{1 / 2} \cdot x_{1}\right) \cdot\left(a_{1}+a_{1}^{*}\right)-b_{1 / 2} \cdot\left(x_{1} \cdot a_{1}\right) \\
& =2\left(b_{1 / 2} \cdot x_{1}\right) \cdot a_{1}-\left(b_{1 / 2} \cdot x_{1}\right) \cdot a_{1}-\left(b_{1 / 2} \cdot a_{1}\right) \cdot x_{1} \\
& =0 \text { by }\left(b_{1}, x_{1 / 2}, a_{1}\right)=0
\end{aligned}
$$




$$
\begin{aligned}
&\left(b_{1 / 2}, x_{1 / 2}, a_{1}+a_{1}^{*}\right)=\left(b_{1 / 2} \cdot x_{1 / 2}\right) \cdot\left(a_{1}+a_{1}^{*}\right)-b_{1 / 2} \cdot\left(x_{1 / 2} \cdot\left(a_{1}+a_{1}^{*}\right)\right) \\
&=\left(b_{1 / 2} \cdot x_{1 / 2}\right) \cdot a_{1}+\left(b_{1 / 2} \cdot x_{1 / 2}\right) \cdot a_{1}^{*}-b_{1 / 2} \cdot\left(x_{1 / 2} \cdot a_{1}\right)-b_{1 / 2} \cdot\left(x_{1 / 2} \cdot a_{1}^{*}\right) \\
&=\left(\left(b_{1 / 2} \cdot a_{1}\right) \cdot x_{1 / 2}\right) \cdot e_{1}+\left(\left(x_{1 / 2} \cdot a_{1}\right) \cdot b_{1 / 2}\right) \cdot e_{1} \\
&+\left(\left(b_{1 / 2} \cdot a_{1}^{*}\right) \cdot x_{1 / 2}\right) \cdot e_{2}+\left(\left(x_{1 / 2} \cdot a_{1}^{*}\right) \cdot b_{1 / 2}\right) \cdot e_{2} \\
&-b_{1 / 2} \cdot\left(x_{1 / 2} \cdot a_{1}\right)-b_{1 / 2} \cdot\left(x_{1 / 2} \cdot a_{1}^{*}\right) \text { by Theorem } 2.4 \\
&=\left(e_{1}-e_{2}\right) \cdot\left(\left(b_{1 / 2} \cdot a_{1}\right) \cdot x_{1 / 2}\right)+\left(e_{2}-e_{1}\right) \cdot\left(\left(b_{1 / 2} \cdot a_{1}^{*}\right) \cdot x_{1 / 2}\right)=0
\end{aligned}
$$

Therefore $J=T+T\left(e_{1}-e_{2}\right)+J_{4 / 2}$ is a Jordan algebra of a nondegenerate symmetric bilinear form on a vector space over the periodic field $T=$ $\left\{a_{11}+a_{11}^{*} \mid a_{11} \in J_{1}\right\}$ with the skew element $e_{1}-e_{2}$ such that $\left(e_{1}-e_{2}\right)^{2}$ $=1$.

LemMA 3.10. Let $J$ be semisimple and $S$ be a periodic Jordan division ring. If $\langle S, k\rangle$ is a periodic field for every invertible skew element $k$, then $J$ is a periodic field.

Proof. We know from Lemma 3.7 that all elements of $J$ are either invertible or nilpotent. Hence by Theorem 2.11, the set of all nilpotent elements forms an ideal $I$. Since $J$ is semisimple, $I=0$. Hence $J$ is a periodic field.

LemMA 3.11. Let $J$ be a Jordan ring with involution and $S$ be a periodic Jordan division ring. If $S=S(+)$ relative to an invertible skew element $k$ and $k^{2}$ is a nonsquare in $S(+)$, the $\langle S, k\rangle$ is a periodic field.

Proof. We know by Lemma 3.8 that $\langle S, k\rangle$ is associative. Since $k^{2}$ is a nonsquare in $S(+),\langle S, k\rangle$ is a field. Hence $\langle S, k\rangle$ is a periodic field by Theorem 3.3.

Lemma 3.12. Let $J$ be semisimple and $S$ be a periodic Jordan division ring. If $k^{2} \neq 0$ is a nonsquare in its corresponding $S(+)$ for all invertible skew elements $k$ and $S \neq S(+)$ for at least one such $k$, then the decomposition is unique in the sense that: If $S=S(+)+S(-)$ is a decomposition relative to an invertible $k$, then it is a decomposition relative to any other invertible skew element.

Proof. Since $J$ is semisimple, $K^{2} \neq 0$. Hence the set of all invertible skew elements is nonempty. Let $S=S(+)+S(-)$ be a decomposition relative to an invertible skew element $k$ such that $S \neq S(+)$ and $S=H(+)+H(-)$ be a decomposition relative to another invertible skew element $k_{1} \in K$. Then either $H(+)=S$ or $[S: H(+)]=2$. But $[S: H(+)]=[S: S(+)]=2 \mathrm{im}$ plies $H(+)=S(+)$ by Lemma 3.5. Hence we assume $H(+)=S$.

Consider $k+k_{1}$ and $k-k_{1}$. Suppose $\left(k+k_{1}\right)^{2}=0$ and $\left(k-k_{1}\right)^{2}$ 
$=0$. We have $k^{2}+k_{1}^{2}=1 / 2\left(\left(k+k_{1}\right)^{2}+\left(k-k_{1}\right)^{2}\right)=0$. Since $-1, k^{2} \in$ $S(+)$, we can apply Lemma 3.6 to find $a, b \in S$ such that $a^{2}=-1$ and $b^{2}=k^{2}$. This implies $k_{1}^{2}=(a \cdot b)^{2}$ and $\left(k_{1}+a \cdot b\right) \cdot\left(k_{1}-a \cdot b\right)=0$. But $\left\langle S, k_{1}\right\rangle$ is a periodic field by Lemma 3.11. Hence $k_{1}= \pm a \cdot b \in S \cap K=0$ contrary to $k_{1} \neq 0$. Without loss of generality, we assume $\left(k+k_{1}\right)^{2} \neq 0$. If $\left\langle S, k+k_{1}\right\rangle$ is not a periodic field, then $s_{-} \cdot\left(k+k_{1}\right)=0$ for any $s_{-} \in$ $S(-)$. This gives us $\left(s_{-}\right) \cdot k=-\left(s_{-}\right) \cdot k_{1}=0$ which contradicts our assumption that $\left\langle S, k_{1}\right\rangle$ is a periodic field. Hence $\left\langle S, k+k_{1}\right\rangle$ is a periodic field. But $\left(s_{-}\right) \cdot\left(k+k_{1}\right)=\left(s_{-}\right) \cdot k_{1}$. Then $k+k_{1}=s_{-}^{-1} \cdot\left(s_{-} \cdot\left(k+k_{1}\right)\right)=s_{-}^{-1}$ - $\left(s_{-} \cdot k_{1}\right)=k_{1}$ contrary to $k \neq 0$. This completes the proof of the above lemma.

LEMMA 3.13. Let $S=S(+)+S(-)$ be the unique decomposition in Lemma

\subsection{Then we have}

(1) $S(-) \cdot K=0$.

(2) $k^{2} \in S(+)$ for all $k \in K$.

(3) $S(+)^{2} \subseteq S(+), S(+) \cdot S(-) \subseteq S(-), S(-)^{2} \subseteq S(+)$.

(4) $\left(s_{1} \cdot s_{2}\right) \cdot k=s_{1} \cdot\left(s_{2} \cdot k\right)$ for all $s_{1}, s_{2} \in S(+), k \in K$.

(5) If $k_{1} \cdot k_{2}=0$, then $\left(s \cdot k_{1}\right) \cdot k_{2}=0$ for all $k_{1}, k_{2} \in K, s \in S(+)$.

(6) $\left(s, k_{1}, k_{2}\right)=0$ for $k_{1}, k_{2} \in K, s \in S(+)$.

(7) $S(+)$ lies in the center of $J$.

Proof. Since $\left(k^{2}, k, k\right)=0, k^{2} \in S(+)$. This proves (2). (3) is clear from Lemma 3.12 and Lemma 3.8. To prove (1) we need only show $s \cdot k=0$ when $s \in S(-), k \in K$ and $k^{2}=0$. We know already that not both $\left(k+k_{1}\right)^{2}=0$ and $\left(k-k_{1}\right)^{2}=0$ for an invertible skew element $k_{1}$. Otherwise $2 k_{1}^{2}=\left(k+k_{1}\right)^{2}+$ $\left(k-k_{1}\right)^{2}=0$. Without loss of generality, assume $\left(k+k_{1}\right)^{2} \neq 0$. Then $s \cdot$ $\left(k+k_{1}\right)=0$ and so $s \cdot k=-s \cdot k_{1}=0$.

To prove (4), we need only show $\left(s_{1}, s_{2}, k\right)=0$ when $k^{2}=0$. But $\left(k+k_{1}\right)^{2}$ and $\left(k-k_{1}\right)^{2}$ are not both equal to zero when $k_{1}^{2} \neq 0$. Assume $\left(k_{1}+k\right)^{2} \neq 0$. Then we have $\left(s_{1} \cdot s_{2}\right) \cdot\left(k_{1}+k\right)=s_{1} \cdot\left(s_{2} \cdot\left(k_{1}+k\right)\right)$ and $\left(s_{1} \cdot s_{2}\right) \cdot k_{1}=s_{1} \cdot\left(s_{2} \cdot k_{1}\right)$. Hence $\left(s_{1} \cdot s_{2}\right) \cdot k=s_{1} \cdot\left(s_{2} \cdot k\right)$.

$$
2\left(\left(s \cdot k_{1}\right) \cdot k_{2}\right) \cdot k_{1}=2\left(s \cdot k_{1}\right) \cdot\left(k_{2} \cdot k_{1}\right)+\left(s \cdot k_{2}\right)
$$

$$
\begin{aligned}
& \cdot k_{1}^{2}-\left(k_{2} \cdot k_{1}^{2}\right) \cdot s \quad \text { by the Jordan identity } \\
= & 0+\left(k_{2} \cdot k_{1}^{2}\right) \cdot s-\left(k_{2} \cdot k_{1}^{2}\right) \cdot s=0 .
\end{aligned}
$$

Thus $\left(s \cdot k_{1}\right) \cdot k_{2} \in S(+) \cap S(-)=0$.

(6) We first show $\left(s, k_{1}, k_{2}\right)=0$ when $k_{2}^{2} \neq 0$. If $k_{1} \cdot k_{2}=0$, then $\left(s, k_{1}, k_{2}\right)=0$ follows from Lemma $3.13(5)$. Hence assume $k_{1} \cdot k_{2} \neq 0$. Let $t=k_{1}-\left[\left(k_{2} \cdot k_{1}\right) \cdot k_{2}^{-2}\right] \cdot k_{2}$. Then $t \cdot k_{2}=0$ by Lemma $3.13(2)$ and so 


$$
s \cdot\left(k_{1}-\left(\left(k_{2} \cdot k_{1}\right) \cdot k_{2}^{-2}\right) \cdot k_{2}\right) \cdot k_{2}=0 .
$$

That is $\left(s \cdot k_{1}\right) \cdot k_{2}=\left(s \cdot\left(k_{2} \cdot k_{1}\right) \cdot k_{2}^{-1}\right) \cdot k_{2} \cdot k_{2}=s \cdot\left(k_{2} \cdot k_{1}\right)$. Similarly $k_{1}^{2} \neq 0$ implies $\left(s, k_{1}, k_{2}\right)=0$. Hence, we assume $k_{1}^{2}=k_{2}^{2}=0$ and $k_{1} \cdot$ $k_{2} \neq 0$. Then $\left(k_{1}+k_{2}\right)^{2} \neq 0$ and so $\left(s, k_{1}, k_{2}\right)=\left(s, k_{1}, k_{1}+k_{2}\right)=0$.

(7) follows immediately from (1) to (6).

LEMMA 3.14. Let $J$ be semisimple with an identity element 1 , and $S$ be simple. Then $J$ is simple or a direct sum of two simples with exchange involution.

Proof. Let $I$ be any *ideal of $J$. Then $I \cap S=(0)$ or $I \cap S=S$. If $I \cap S=(0)$, then $I \subseteq K$ and so $I^{2} \subseteq K \cap S=(0)$. Thus $I=0$ from the fact that $J$.is semisimple. If $I \cap S=S$, then $1 \in I$ and $I=J$. Hence $J$ is *-simple. If $J$ is not simple, let $I$ be any nonzero ideal of $J$, then $I+I^{*}$ and $I \cap I^{*}$ are ${ }^{*}$ ideals. Therefore $I+I^{*}=J$ and $I \cap I^{*}=(0)$ if $J$ is not simple.

THEOREM 3.15. Let $J$ be semisimple and $S$ be a periodic Jordan division ring. Then $J$ is one of the following:

(1) a periodic field;

(2) a direct sum of two periodic fields with exchange involution,

(3) a Jordan algebra of a nondegenerate symmetric bilinear form $f$ on a vector space over a subfield of $S$.

Proof. We know from Lemma 3.14 that $J$ is ${ }^{*}$-simple. If $J$ is a direct sum of two simples interchanged by the involution, then $J$ is a direct sum of two periodic fields with exchange involution. Hence, we assume $J$ is simple.

Let $k$ be any invertible skew element. If $k^{2}$ is a square in its corresponding $S(+)$, then $J$ is a Jordan algebra of a nondegenerate symmetric bilinear form over a subfield of $S$ by Lemma 3.9. Hence assume $k^{2}$ is a nonsquare in its corresponding $S(+)$ for all invertible skew elements $k$. Then two cases arise. Either $S=S(+)$ for all invertible skew elements or $S \neq S(+)$ for at least one invertible skew element. $J$ is a periodic field in case one by Lemma 3.10 and Lemma 3.11. If $S \neq S(+)$, then by Lemma 3.12 and Lemma 3.13, we can decompose $S=S(+)+S(-)$ such that $S(+)$ lies in the center of $J, S(-) \cdot$ $K=0$ and $K^{2} \subseteq S(+)$. This means $J$ is a Jordan algebra of a symmetric bilinear form over $S(+)$.

4. Classification of *-prime Jordan rings when $S$ is periodic and contains at least two orthogonal idempotents. A nonassociative ring is said to be * ${ }^{*}$-prime if given by any two ${ }^{*}$ ideals $B$ and $C$ such that $B C=0$, then $B=0$ or $C=0$. We need the following theorem. 
THEOREM 4.1 (BRITTEN [1]). Let $R$ be a nonassociative ring with involution ${ }^{*}$. Then $R$ is *-prime if and only if $R$ is prime or $R$ contains a prime ideal $P$ such that $P \cap P^{*}=0$.

In this section, we show any *-prime Jordan ring $J$ with periodic symmetric elements contains no more than four orthogonal idempotents. Then we classify $J$ according to the number of orthogonal idempotents that $S$ contains. The following two theorems were done by Osborn [3] when $J$ is a periodic Jordan ring.

THEOREM 4.2. Let $J$ be a Jordan ring with involution ${ }^{*}$ and $S$ be periodic. Let $e$ and $f$ be two symmetric orthogonal idempotents. If $b \in$ $J_{1 / 2}(e) \cap J_{1 / 2}(f)$ is skew (or symmetric) such that $b^{n}=e+f$ for some positive integer $n$ greater than 1 , then for any symmetric idempotent $u \in J_{1}(e)$, there exists an integer $m$ such that $\left[(2 b \cdot u)^{2} \cdot u\right]^{m}=u$.

Proof. Let $B$ be the Jordan subring generated by $b$ and $u$. $B$ has the identity element $e+f$, so by Shirshov's theorem, $B$ is isomorphic to the set of symmetric elements of an associative ring $C$ with involution. Let $C=C_{11}+$ $C_{10}+C_{01}+C_{00}$ be the Peirce decomposition relative to the idempotent $u$. Since $b \in J_{1 / 2}(e) \subseteq J_{1 / 2}(u)+J_{0}(u)$ we have $b \in C_{10}+C_{01}+C_{00}$ and $u b u=0$ where juxtaposition indicates multiplication in $C$ and

$$
(2 b \cdot u)^{2} \cdot u=(b u+u b)^{2} \cdot u=\left(b u b+u b^{2} u\right) \cdot u=u b^{2} u .
$$

Hence $\left[(2 b \cdot u)^{2} \cdot u\right]^{m}=u$ for some $m$ if and only if $\left(u b^{2} u\right)^{m}=u$.

We now show $u b^{2} u \neq 0$. If $u b^{2} u=0$ then $(b u b)^{2}=0$. But $b u b$ is a periodic symmetric element of $J$, so $b u b=0$. This implies $b^{n} u b^{n}=$ $(e+f) u(e+f)=u=0$ which contradicts $u \neq 0$.

Since $u b^{2} u$ is a nonzero ${ }^{*}$ symmetric element of $J$, we have $\left(u b^{2} u\right)^{m+1}$ $=u b^{2} u$ for some $m$ greater than zero. We now show $\left(u b^{2} u\right)^{m}=u$. Let

$$
t=2\left(u-\left(u b^{2} u\right)^{m}\right) \cdot b=u b+b u-\left(u b^{2} u\right)^{m} b-b\left(u b^{2} u\right)^{m} .
$$

We calculate that

$$
\begin{aligned}
t^{2}= & u b^{2} u+b u b+\left(u b^{2} u\right)^{2 m+1}+b\left(u b^{2} u\right)^{2 m} b \\
& -2\left(u b^{2} u\right)^{m+1}-2 b\left(u b^{2} u\right)^{m} b \\
= & b u b-b\left(u b^{2} u\right)^{m} b, \\
t^{4}= & b u b^{2} u b-2 b\left(u b^{2} u\right)^{m+1} b+b\left(u b^{2} u\right)^{2 m+1} b=0 .
\end{aligned}
$$

But any symmetric element is periodic, so $t^{2}=0$. Then 


$$
u U_{b}=\left(u b^{2} u\right)^{m} U_{b}, \quad u U_{b}^{n}=\left(u b^{2} u\right)^{m} U_{b}^{n} .
$$

Therefore $u=\left(u b^{2} u\right)^{m}$.

THEOREM 4.3. Let $J$ be a Jordan ring with involution ${ }^{*}$ and $S$ be periodic. Let $e$ and $f$ be two symmetric orthogonal idempotents. If $b \in$ $J_{1 / 2}(e) \cap J_{1 / 2}(f)$ is skew (or symmetric) such that $b^{n}=e+f$ for some positive integer $n$ greater than 1, then for any symmetric idempotent $u \in J_{1}(e)$, there exists a positive integer $l$ such that $(2 b \cdot u)^{l}=u+v$ where $v$ is a symmetric idempotent in $J_{1}(f)$, also $2 b \cdot u \in J_{1 / 2}(u) \cap J_{1 / 2}(v)$.

Proof. Since $J_{1 / 2}(e) \subseteq J_{1 / 2}(u)+J_{0}(u)$, we have

$$
2 b \cdot u \in\left(J_{1 / 2}(u)+J_{0}(u)\right) J_{1}(u) \subseteq J_{1 / 2}(u) J_{1}(u) \subseteq J_{1 / 2}(u) .
$$

Similarly $2 b \cdot u \in J_{1 / 2}(f)$. This implies $2 b \cdot u \in J_{1 / 2}(u) \cap J_{1 / 2}(f)$. But $\left[(2 b \cdot u)^{2} \cdot u\right]^{m}=u \neq 0$ for some integer $m$ and so $(2 b \cdot u)^{2} \neq 0$. We can find a positive integer $l$ such that $(2 b \cdot u)^{l}=u_{1}+v$ where $u_{1} \in J_{1}(u)$, and $v \in$ $J_{1}(f)$ are idempotents. Then $u_{1}+v=(2 b \cdot u)^{2 l}=\left[(2 b \cdot u)^{2}\right]^{l}$. Since $(2 b \cdot u)^{2} \in J_{1}(u)+J_{1}(f),\left[(2 b \cdot u)^{2} \cdot u\right]^{l}=u_{1}$. Hence $u_{1}=u_{1}^{m}=$ $\left[(2 b \cdot u)^{2} \cdot u\right]^{l m}=u^{l}=u$.

To prove the last part we know $b \in J_{1 / 2}(f) \subseteq J_{1 / 2}(v)+J_{1}(v)$ from $v \in$ $J_{1}(f)$. Thus $2 b \cdot u \in\left(J_{1 / 2}(v)+J_{1}(v)\right) J_{0}(v) \subseteq J_{1 / 2}(v)$ and so $2 b \cdot u \in J_{1 / 2}(u) \cap$ $J_{1 / 2}(v)$.

We mention the following theorem of Jacobson which will be used in the proof of the next theorem.

THEOREM 4.4 (JACOBSON [1, p. 122]). Let $e_{1}, e_{2}, e_{3}$ be pairwise orthogonal idempotents in a Jordan ring $A$. If $e_{1}, e_{2}$ are connected by an element $b \in J_{1 / 2}\left(e_{1}\right) \cap J_{1 / 2}\left(e_{2}\right), e_{2}$ and $e_{3}$ are connected by $c \in J_{1 / 2}\left(e_{2}\right) \cap J_{1 / 2}\left(e_{3}\right)$, then $e_{1}$ and $e_{3}$ are connected by $b \cdot c \in J_{1 / 2}\left(e_{1}\right) \cap J_{1 / 2}\left(e_{3}\right)$.

TheOREM 4.5. Let $J$ be *-prime and $S$ be periodic. Then $J$ has an identity element.

Proof. Let $e$ be any idempotent in $S$. If $e$ is the only idempotent, then $S$ is a periodic field and by Theorem 3.1,e is the identity of $J$. Hence assume $S_{0}(e) \neq 0$. Let $J=J_{1}(e)+J_{1 / 2}(e)+J_{0}(e)$ be the Peirce decomposition relative to the idempotent $e$. Since $J$ is ${ }^{*}$-prime, $\left(J_{1 / 2}(e)\right)^{2} \neq 0$, we can find $x \in J_{1 / 2}(e)$ which is symmetric or skew depending on $S_{1 / 2}(e) \neq 0$ or $S_{1 / 2}(e)=0$, such that $x^{n}=e_{1}+e_{2}$ where $e_{1}$ and $e_{2}$ are symmetric orthogonal idempotents. If $e_{1}+e_{2}$ is not the identity element of $S$, we now show that we can find three orthogonal symmetric connected idempotents. 
Consider $J=J_{1}\left(e_{1}+e_{2}\right)+J_{1 / 2}\left(e_{1}+e_{2}\right)+J_{0}\left(e_{1}+e_{2}\right)$ which is equal to

$$
\begin{aligned}
J= & J_{1}\left(e_{1}\right)+J_{1}\left(e_{2}\right)+J_{1 / 2}\left(e_{1}\right) \cap J_{1 / 2}\left(e_{2}\right) \\
& +J_{0}\left(e_{1}\right) \cap J_{1 / 2}\left(e_{2}\right)+J_{1 / 2}\left(e_{1}\right) \cap J_{0}\left(e_{2}\right)+J_{0}\left(e_{1}\right) \cap J_{0}\left(e_{2}\right)
\end{aligned}
$$

and so $J_{1 / 2}\left(e_{1}+e_{2}\right)=J_{1 / 2}\left(e_{1}\right) \cap J_{0}\left(e_{2}\right)+J_{1 / 2}\left(e_{2}\right) \cap J_{0}\left(e_{1}\right), J_{0}\left(e_{1}+e_{2}\right)=$ $J_{0}\left(e_{1}\right) \cap J_{0}\left(e_{2}\right)$. (See Osborn [3, p. 224].) Let $b=b_{1}+b_{2} \in J_{1 / 2}\left(e_{1}+e_{2}\right)$ where $b_{1} \in J_{1 / 2}\left(e_{1}\right) \cap J_{0}\left(e_{2}\right), b_{2} \in J_{1 / 2}\left(e_{2}\right) \cap J_{0}\left(e_{1}\right)$ and $b^{2} \neq 0$. We can assume $b$ is either symmetric or skew. But $b^{2}=b_{1}^{2}+b_{2}^{2}+2 b_{1} \cdot b_{2}$ is symmetric and $2 b_{1} \cdot b_{2} \in J_{1 / 2}\left(e_{1}\right) \cap J_{1 / 2}\left(e_{2}\right)$. Therefore $b_{1}^{2} \neq 0$ or $b_{2}^{2} \neq 0$. Otherwise $b^{2} \in J_{1}\left(e_{1}+e_{2}\right)$ and $\left(\left(e_{1}+e_{2}\right) \cdot b^{2}\right) \cdot b=b^{3}=\left(\left(e_{1}+e_{2}\right) \cdot b\right) \cdot b^{2}=2^{-1} b^{3}$ give $b^{4}=0$ which contradicts the fact that $b^{2}$ is a nonzero periodic symmetric element. We assume $b_{1}^{2} \neq 0$. Then there exists a positive integer $n$ such that $b_{1}^{2 n}=u+u_{1}$ where $u \in J_{1}\left(e_{1}\right)$ and $u_{1} \in J_{0}\left(e_{1}\right) \cap J_{0}\left(e_{2}\right)$ are symmetric orthogonal idempotents. Applying Theorem 4.3, $(2 x \cdot u)^{l}=u+u_{2}$ for some positive integer $l$ where $u_{2}$ is a symmetric idempotent in $J_{1}\left(e_{2}\right)$. Hence $u$, $u_{1}, u_{2}$ are three symmetric orthogonal connected idempotents.

It can be shown by induction that

$$
\begin{aligned}
J_{1 / 2}\left(e_{1}+e_{2}+\cdots+e_{n}\right)= & J_{y_{2}}\left(e_{1}\right) \cap \cdots \cap J_{0}\left(e_{n}\right) \\
& +J_{0}\left(e_{1}\right) \cap J_{y_{1}}\left(e_{2}\right) \cap \cdots \cap J_{0}\left(e_{n}\right)+\cdots \\
& +J_{0}\left(e_{1}\right) \cap \cdots \cap J_{0}\left(e_{n-1}\right) \cap J_{y_{2}}\left(e_{n}\right)
\end{aligned}
$$

and

$$
J_{0}\left(e_{1}+\cdots+e_{n}\right)=J_{0}\left(e_{1}\right) \cap \cdots \cap J_{0}\left(e_{n}\right)
$$

for arbitrary $n$ orthogonal idempotents $e_{1}, e_{2}, \cdots, e_{n}$. Then by the same argument as in the last paragraph, we can find five orthogonal connected symmetric idempotents $\left\{v_{1}, v_{2}, \cdots, v_{5}\right\}$ if we assume $S$ has no identity element. We now prove this cannot happen.

Suppose $v_{1}$ and $v_{2}$ are connected by a symmetric element $b_{12}$. Then $v_{2}$ and $v_{3}$ are connected by a skew element $b_{23}, v_{3}$ and $v_{4}$ are connected by a symmetric element $b_{34} \cdot v_{4}$ and $v_{5}$ are connected by a skew element $b_{45}$ by Theorem 2.9. But this implies $v_{2}$ and $v_{5}$ are connected by the symmetric element $\left(b_{23} \cdot b_{34}\right) \cdot b_{45}$ which contradicts Theorem 2.9.

Similarly if $v_{1}$ and $v_{2}$ are connected by a skew, we get a contradiction. Hence $S$ has an identity 1 and by Theorem3.1 $J$ has the same identity 1 .

Theorem 4.6. Let $J$ be *-prime and $S$ be periodic. Then $S$ contains no more than four orthogonal idempotents.

Proof. We have shown in the previous theorem that $J$ has the identity 
1. Suppose $S$ contains more than four orthogonal idempotents. Then there exists five orthogonal idempotents such that $1=e_{1}+e_{2}+e_{3}+e_{4}+e_{5}$. If $J_{1 / 2}\left(e_{1}\right) \cap J_{1 / 2}\left(e_{i}\right)=0$ for all $i=2,3,4,5$, then

$$
J=J_{1}\left(e_{1}\right)+J_{1}\left(e_{2}+e_{3}+e_{4}+e_{5}\right)
$$

which contradicts our assumption that $J$ is * ${ }^{*}$-prime. Hence $J_{1 / 2}\left(e_{1}\right) \cap$ $J_{y_{2}}\left(e_{i}\right) \neq 0$ for at least one $i$. For convenience, assume $J_{1 / 2}\left(e_{1}\right) \cap$ $J_{1 / 2}\left(e_{2}\right)=J_{12} \neq 0$. Since $J_{1}\left(e_{1}+e_{2}\right)$ is semisimple by Theorem $2.5, J_{1 / 2}^{2} \neq 0$. Then we can find $b_{12} \in J_{12}$ which is skew or symmetric such that $b_{12}^{n}=$ $u_{1}+u_{2}$ where $u_{i} \in J_{1}\left(e_{i}\right)(i=1,2)$ and so $u_{1}, u_{2}, e_{3}, e_{4}, e_{5}$ are five orthog. onal idempotents. We can assume $1=u_{1}+u_{2}+e_{3}+e_{4}+e_{5}$ without loss of generality. Then $J_{1 / 2}\left(u_{1}+u_{2}\right) \cap J_{1 / 2}\left(e_{3}+e_{4}+e_{5}\right) \neq 0$. Assume $J_{1 / 2}\left(u_{2}\right) \cap$ $J_{1 / 2}\left(e_{3}\right) \neq 0$. By the same argument, there exists $b_{23} \in J_{1 / 2}\left(u_{2}\right) \cap J_{1 / 2}\left(e_{3}\right)$ such that $b_{23}^{l}=v_{2}+v_{3}$ where $v_{2} \in J_{1}\left(u_{2}\right)$ and $v_{3} \in J_{1}\left(e_{3}\right)$ are symmetric idempotents. By Theorem 4.3, $\left(2 b_{12} \cdot v_{2}\right)^{m}=v_{2}+v_{1}$ where $v_{1} \in J_{1}\left(u_{1}\right)$ is a symmetric idempotent. Thus $\left\{v_{1}, v_{2}, v_{3}, e_{4}, e_{5}\right\}$ are five orthogonal idempotents and $v_{1}, v_{2}, v_{3}$ are connected. By continuing the above process, we finally get five orthogonal symmetric connected idempotents which by the proof of Theorem 4.5 is impossible.

Lemma 4.7. Let $J$ be *-prime and $1=e_{1}+e_{2}+e_{3}$ where $e_{i}$ are symmetric orthogonal idempotents. If $J_{1}\left(e_{1}\right)$ and $J_{1}\left(e_{2}\right)$ are periodic fields, $J_{1 / 2}\left(e_{1}+e_{2}\right) \subseteq K$, then $J_{1}\left(e_{1}+e_{2}\right) \subseteq S$.

Proof. Let $W=\left\{w \in J_{1}\left(e_{1}+e_{2}\right) \mid w \cdot J_{1 / 2}\left(e_{1}+e_{2}\right)=0\right\}$. Then

$$
\left(K \cap J_{1}\left(e_{1}+e_{2}\right)\right) \cdot J_{1 / 2}\left(e_{1}+e_{2}\right) \subseteq S \cap J_{1_{1}}\left(e_{1}+e_{2}\right)=0
$$

and so $K \cap J_{1}\left(e_{1}+e_{2}\right) \subseteq W$. To show that $W$ is an ideal of $J_{1}\left(e_{1}+e_{2}\right)$, let $w \in W, a \in J_{1}\left(e_{1}+e_{2}\right)$. Then

$$
(w \cdot a) \cdot J_{1 / 2}\left(e_{1}+e_{2}\right)=w \cdot\left(a \cdot J_{1 / 2}\left(e_{1}+e_{2}\right)\right)+a \cdot\left(w \cdot J_{1 / 2}\left(e_{1}+e_{2}\right)\right)=0
$$

and so $w \cdot a \in W$. If there exist $0 \neq s=s_{1}+s_{1 / 2}+s_{0} \in S \cap W$ where $s_{i} \in$ $S_{i}\left(e_{1}\right)$, then $s_{i}(i=1,1 / 2,0) \in S \cap W$. This will imply $e_{1}+e_{2} \in W$ or $e_{i}$ $(i=1,2) \in W$. Thus $S \cap W=0$ and $\left[K \cap J_{1}\left(e_{1}+e_{2}\right)\right]^{2}=W^{2}=0$. But $J_{1}\left(e_{1}+e_{2}\right)$ is semisimple and so $K \cap J_{1}\left(e_{1}+e_{2}\right)=0$.

LEMMA 4.8. Let $A=\left\{\left[\begin{array}{cc}a & b^{*} \mu \\ b & c\end{array}\right] \mid a, b, c, \mu \in D, \mu^{*}=\mu, a^{*}=a, c^{*}=c\right\}$ where $D$ is $a^{*}$-simple associative ring from the following list:

(1) a periodic field with involution,

(2) a direct sum of two periodic fields interchanged by the involution, 
(3) a $2 \times 2$ matrix algebra over a periodic field with standard involution,

(4) a Cayley-Dickson algebra over a periodic field with standard involution, and the multiplication in $A$ is defined as

$$
\begin{aligned}
& {\left[\begin{array}{cc}
a & b^{*} \mu \\
b & c
\end{array}\right] \cdot\left[\begin{array}{cc}
m & p^{*} \mu \\
p & n
\end{array}\right]} \\
& \quad=\left[\begin{array}{cc}
a m+p b^{*} \mu & 2^{-1}(b m+b n+p a+p c)^{*} \mu \\
2^{-1}(b m+b n+p a+p c) & b p^{*} \mu+c n
\end{array}\right] .
\end{aligned}
$$

If $A$ is periodic, then $D$ is a periodic field with identity involution and $-\mu$ is a nonsquare in $D$.

Proof. If $D$ is of type (2), (3) or (4), then there exists an element $b$ such that $b \bar{b}=0$. Then $\left[\begin{array}{cc}0 & \bar{b} \mu \\ b & 0\end{array}\right]^{2}=0$ contrary to the assumption that $A$ is periodic. If $D$ is a periodic field with nonidentity involution and if $k$ is any nonzero skew element, then by Theorem 2.8, there exist symmetric elements $a, b, c$ such that $a^{2}-b^{2} k^{2}+c^{2} \mu=0$. Let $w=a c^{-1}-b c^{-1} k$. We have $w \bar{w}+\mu=0$. Therefore $\left[\begin{array}{cc}\mu & \bar{w} \mu \\ w & -\mu\end{array}\right]^{2}=0$. Hence $D$ is a periodic field with identity involution and $-\mu$ is a nonsquare in $D$.

THEOREM 4.9. Let $J$ be *-prime and $S$ be periodic. If $S$ contains exactly four orthogonal idempotents, then $J$ is a $4 \times 4$ Jordan matrix algebra over a periodic field.

Proof. Let the four orthogonal idempotents that $S$ contains be $e_{1}, e_{2}$, $e_{3}$, and $e_{4}$. Then $S_{1}\left(e_{i}\right)(i=1,2,3,4)$ are periodic fields and $1=e_{1}+$ $e_{2}+e_{3}+e_{4}$. Otherwise $S$ contains more than four orthogonal idempotents. Then the idempotents $e_{i}$ are connected. Changing the subscripts and by Theorem 2.9, we can assume that $e_{1}$ and $e_{2}$ are connected in $S, e_{2}$ and $e_{3}$ are connected by a skew, $e_{3}$ and $e_{4}$ are connected by a symmetric element. Then all elements in $J_{1 / 2}\left(e_{1}+e_{2}\right) \cap J_{1 / 2}\left(e_{3}+e_{4}\right)=J_{13}+J_{14}+J_{23}+J_{24}$ are skew. Hence by Lemma $4.8, J_{1}\left(e_{1}+e_{2}\right)=S_{1}\left(e_{1}+e_{2}\right), J_{1}\left(e_{3}+e_{4}\right)=$ $S_{1}\left(e_{3}+e_{4}\right)$.

Then applying Theorem 2.14, Theorem 2.3 and Theorem $2.12, J$ is isomorphic to a $4 \times 4$ Jordan matrix algebra over a $j$ simple associative algebra $D$ with involution $j: x \rightarrow \bar{x}$ where $D$ is one of the following:

(1) a periodic field with involution,

(2) a direct sum of two periodic fields interchanged by the involution,

(3) a $2 \times 2$ matrix algebra over a periodic field with standard involution, That is: 


$$
\begin{gathered}
\left.J \sim\left\{\begin{array}{cccc}
a & \bar{b} u & \bar{c} v & \bar{d} w \\
b & e & u^{-1} \bar{f} v & u^{-1} \bar{g} w \\
c & f & p & v^{-1} \bar{h} w \\
d & g & h & k
\end{array}\right] \mid a, b, c, d, e, f, g, h, p, k \in D\right\}, \\
\bar{u}=u, \bar{v}=v, \bar{w}=w, \bar{a}=a, \bar{p}=p, \bar{k}=k, \bar{e}=e .
\end{gathered}
$$

But $\left.\left\{\begin{array}{cc}a & \bar{b} u \\ b & e\end{array}\right] \mid a, b, e, u \in E, \bar{a}=a, \bar{e}=e, \bar{u}=u\right\}$ is periodic and so $D$ is a periodic field with the identity involution and $-u$ is a nonsquare element in $D$ by Lemma 4.8. This completes the proof of this theorem.

THEOREM 4.10. Let $J$ be ${ }^{*}$-prime and $S$ be periodic. If $S$ contains exactly three orthogonal idempotents, then $J$ is a $3 \times 3$ Jordan matrix algebra over a periodic field.

Proof. Let the idempotents that $S$ contains be $e_{1}, e_{2}$ and $e_{3}$. Then $S_{1}\left(e_{i}\right)(i=1,2,3)$ are periodic fields and $1=e_{1}+e_{2}+e_{3}$. We now show that $\left\{e_{1}, e_{2}, e_{3}\right\}$ are connected. Suppose $e_{1}$ and $e_{2}$ are not connected, then $J_{12}=0$. Since connectedness of idempotents is a transitive relation, $J_{23}=0$ or $J_{13}=0$. Assume $J_{13}=0$. Then $J=J_{11}+\left(J_{22}+J_{23}+J_{33}\right)$ contrary to our assumption on $J$, similarly for any other two idempotents which are not connected. Hence $e_{1}, e_{2}, e_{3}$ are connected. Changing the subscripts if necessary, we assume that $e_{1}$ and $e_{2}$ are connected by a symmetric element, $e_{2}$ and $e_{3}$ are connected by a skew element. Then by Theorem $2.9, J_{23} \subseteq K$ and by Lemma 4.7, $J_{1}\left(e_{1}+e_{2}\right)$ $=S_{1}\left(e_{1}+e_{2}\right)$. Applying Theorem 2.3, Theorem 2.12 and Theorem 2.14, $J$ is isomorphic to a $3 \times 3$ Jordan matrix algebra over a $j$ simple alternative algebra $(D, j)$ with involution $j$ where $D$ is one of the following: (i) a periodic field with involution, (ii) a direct sum of two periodic fields interchanged by the involution, (iii) a $2 \times 2$ matrix algebra over a periodic field with the standard involution, (iv) a CayleyDickson algebra over a periodic field with the standard involution. That is

$$
\left\{\left[\begin{array}{ccc}
a & \bar{b} u & \bar{c} v \\
b & d & u^{-1} \bar{e} v \\
c & e & f
\end{array}\right] \mid \bar{a}=a, \bar{d}=d, \bar{f}=f, \bar{u}=u, \bar{v}=v\right\}
$$

But $\left.\left\{\begin{array}{cc}a & \bar{b} \\ b & d\end{array}\right] \mid a, b, d, u \in D, \bar{a}=a, \bar{d}=d, \bar{u}=u\right\}$ is periodic. Thus $D$ is a periodic field with the identity involution by Lemma 4.7.

THEOREM 4.11. Let $J$ be $e^{*}$-prime and $S$ be periodic. If $S$ contains exactly two orthogonal idempotents, then $J$ is one of the following:

(1) a direct sum of two simple periodic Jordan algebras of capacity two 
interchanged by the involution,

(2) a Jordan algebra of a nondegenerate symmetric bilinear form on a vector space over a periodic field,

(3) a $4 \times 4$ Jordan matrix algebra over a periodic field.

The proof of the above theorem is quite complicated. For convenience, we divide the proof into several steps.

Assertion 1. $S$ is either a simple periodic Jordan ring of capacity two or $S$ is a direct sum of two periodic fields.

Proof. Let $e_{1}$ and $e_{2}$ be the two orthogonal idempotents that belong to $S$. If $e_{1}$ and $e_{2}$ are connected in $S$, then by Theorem $2.9 S=S_{1}\left(e_{1}+e_{2}\right)+$ $S_{0}\left(e_{1}+e_{2}\right)$. Thus $S=S_{1}\left(e_{1}+e_{2}\right)$ by the assumption on $S$. Let $I$ be any ideal of $S$ and $0 \neq s=s_{1}+s_{1 / 2}+s_{0} \in I$ where $s_{i} \in S_{i}\left(e_{1}\right)$. Then $s_{i}(i=1,1 / 2,0)$ $\in I$ and so $1 \in I$ or $e_{i} \in I$ for $i=1$, or $i=2$. This implies $2^{-1} x_{1 / 2}=$ $e_{i} \cdot x_{1 / 2}$ for any $x_{1 / 2} \in S_{1 / 2}\left(e_{1}\right)$. Since $S_{1}\left(e_{i}\right)(i=1,2)$ are periodic fields, $x_{1 / 2}^{n}=e_{1}+e_{2} \in I$ and $I=S$.

If $e_{1}$ and $e_{2}$ are not connected, then $S_{1}\left(e_{i}\right)(i=1,2)$ are periodic fields and $S=S_{1}\left(e_{1}\right)+S_{1}\left(e_{2}\right)$.

Assertion 2. If $S$ is a direct sum of two periodic fields, then $S_{1}\left(e_{1}\right)=$ $J_{1}\left(e_{1}\right), S_{1}\left(e_{2}\right)=J_{1}\left(e_{2}\right)$ and $J$ is a Jordan algebra of a nondegenerate symmetric bilinear form on a vector space over a periodic field.

Proof. Let $k \in K \cap J_{1}\left(e_{1}\right)$. Then by Theorem $2.4, k \cdot J_{1 / 2}\left(e_{1}\right) \subseteq S \cap$ $J_{1 / 2}\left(e_{1}\right)=0$ and $k^{2} \cdot J_{1 / 2}\left(e_{1}\right) \subseteq 2 k \cdot J_{1 / 2}\left(e_{1}\right)=0$. If $k^{2} \neq 0, k^{2}$ has an inverse $s \in J_{1}\left(e_{1}\right)$ such that $s \cdot k^{2}=e_{1}$ and by Theorem 2.4

$$
e_{1} \cdot J_{1 / 2}\left(e_{1}\right)=\left(s \cdot k^{2}\right) J_{1 / 2}\left(e_{1}\right) \subseteq s \cdot\left(k^{2} \cdot J_{1 / 2}\left(e_{1}\right)\right)+k^{2} \cdot\left(s \cdot J_{1 / 2}\left(e_{1}\right)\right)=0 .
$$

Thus $J_{1 / 2}\left(e_{1}\right)=0$ and so $J=J_{1}\left(e_{1}\right)+J_{0}\left(e_{1}\right)$ which contradicts our assumption that $J$ is *-prime. Hence $k^{2}=0$ and $K \cap J_{1}\left(e_{1}\right)$ is a nilpotent ideal of $J_{1}\left(e_{1}\right)$. But by Theorem 2.5, $J_{1}\left(e_{1}\right)$ is semisimple, so $K \cap J_{1}\left(e_{1}\right)=0$. Therefore $J_{1}\left(e_{1}\right)=S_{1}\left(e_{1}\right)$. Similarly, $J_{1}\left(e_{2}\right)=S_{1}\left(e_{2}\right)$.

Furthermore, $\left(J_{1 / 2}\left(e_{1}\right)\right)^{2} \neq 0$. Otherwise, $J_{1 / 2}\left(e_{1}\right)$ is a nilpotent ideal of $J$ and the semisimplicity of $J$ will imply $J_{1 / 2}\left(e_{1}\right)=0$. Thus we can find an element $x \in J_{1 / 2}\left(e_{1}\right)$ such that $x^{n}=e_{1}+e_{2}$ for some positive integer $n . J$ is then a simple Jordan algebra of capacity two. Applying Theorem $2.13 J$ is either a Jordan algebra of a nondegenerate symmetric bilinear form $f$ on a vector space over a field $T$, or a Jordan matrix algebra $H\left(D_{2}, J_{n}\right)$ where $D$ is one of the following: (i) a direct sum of two noncommutative associative division algebras with the exchange involution, (ii) a noncommutative division algebra which is not a quaternion algebra over its center with $j$ the standard involution.

But if $J$ is isomorphic to $H\left(D_{2}, J_{n}\right)$, then the set of $j$ symmetric elements 
forms a periodic field and $D$ is either a periodic field or a direct sum of two periodic fields. This contradicts the fact that $D$ is not commutative. Thus $J$ is a Jordan algebra of a nondegenerate symmetric bilinear form $f$ on a vector space over $T$. Since $T \subseteq J_{1}\left(e_{1}\right)+J_{0}\left(e_{1}\right), T$ is a periodic field.

If $S$ is a simple periodic Jordan algebra of capacity two, then by Theorem 3.1, $J$ has the identity 1, and by Lemma $3.14 J$ is *-simple. But if $J$ is a direct sum of two simple ideals interchanged by the involution, then $J$ is a Jordan algebra of type (1) in Theorem 4.10. Otherwise $J$ is simple.

Assertion 3. Let $J$ be simple and the center of $J$ be $Z$. If $Z \nsubseteq S$, then $J$ is a three-dimensional Jordan algebra of a nondegenerate symmetric bilinear form on a vector space over a periodic field.

Proof. Since $Z \nsubseteq S$, we can find a nonzero skew element $k$ in $Z$. But $\left(k_{1} \cdot k^{-1}\right) \cdot k=k_{1}$ for any skew element $k_{1}$, so $J=S+S \cdot k$. Let $C$ be the center of $S$. From $J=S+S \cdot k$ and $k \in Z$, it follows that $C=S \cap Z$. But, by Theorem 2.10, $S=C+C \cdot\left(e_{2}-e_{1}\right)+C \cdot u_{1 / 2}$ where $u_{1 / 2} \in S_{1 / 2}\left(e_{1}\right)$ and $u_{1 / 2}^{2} \in C$. Thus

$$
\begin{aligned}
J & =S+S \cdot k=(C+C \cdot k)+(C+C \cdot k) \cdot\left(e_{2}-e_{1}\right)+(C+C \cdot k) \cdot u_{1 / 2} \\
& =Z+Z \cdot\left(e_{2}-e_{1}\right)+Z \cdot u_{1 / 2}
\end{aligned}
$$

and by Theorem $3.3, Z$ is a periodic field.

Hence, from now on we assume (i) $J$ is simple with the identity element 1, (ii) $S$ is a simple periodic Jordan algebra of capacity 2, (iii) $Z \subseteq S$. Since the sets of symmetric elements of the semisimple Jordan algebras $J_{1}\left(e_{i}\right) \quad(i=$ $1, e)$ form periodic fields, $J_{1}\left(e_{i}\right)(i=1,2)$ are the Jordan algebras of the following types:

(a) a periodic field,

(b) a direct sum of two periodic fields with exchange involution,

(c) a Jordan algebra of a nondegenerate symmetric bilinear form on a vector space over a periodic field.

We have the following possibilities:

Case (1) The $J_{1}\left(e_{i}\right)$ are periodic fields (same as Assertion 2).

Case (2) $J_{1}\left(e_{1}\right)$ is a direct sum of two periodic fields and $J_{1}\left(e_{2}\right)$ is a periodic field.

Case (3) Both $J_{1}\left(e_{1}\right)$ and $J_{1}\left(e_{2}\right)$ are of the same type (b).

Case (4) $J_{1}\left(e_{1}\right)$ is a direct sum of two periodic fields and $J_{1}\left(e_{2}\right)$ is of the type (c).

Case (5) $J_{1}\left(e_{1}\right)$ is a periodic field and $J_{1}\left(e_{2}\right)$ is of the type (c).

Case (6) Both $J_{1}\left(e_{1}\right)$ and $J_{1}\left(e_{2}\right)$ are of the same type (c).

Assertion 4. Case (2) is impossible.

Proof. In Case (2), there exist three orthogonal idempotents $1=a+b+c$ such that $a^{*}=b, a+b=e_{1}, c=c^{*}=e_{2}$. If we let $J=J_{11}+J_{22}+J_{33}+$ 
$J_{12}+J_{13}+J_{23}$ be the Peirce decomposition of $J$ relative to $a, b$, and $c$, such that

$$
\begin{array}{lll}
J_{11}=J_{1}(a), & J_{22}=J_{1}(b), & J_{33}=J_{1}(c), \\
J_{12}=J_{1 / 2}(a) \cap J_{1 / 2}(b), & J_{23}=J_{1 / 2}(b) \cap J_{1 / 2}(c), & J_{13}=J_{1 / 2}(a) \cap J_{1 / 2}(c) ;
\end{array}
$$

then $J_{12}=0$ and $J_{i i}(i=1,2,3)$ are periodic fields. Since $J_{1 / 2}(a+b) \cap$ $J_{1 / 2}(c) \supseteq S_{1 / 2}\left(e_{1}\right) \cap S_{1 / 2}\left(e_{2}\right) \neq 0$, we have $J_{13}+J_{23} \neq 0$. But $J_{13} \neq 0$ if and only if $J_{23}=\left(J_{13}\right)^{*} \neq 0$. Thus $J_{13} \neq 0$ and $J_{23} \neq 0$. If $J_{13}^{2}=0$, then $J_{13}$ is a nilpotent ideal of the semisimple Jordan algebra $J_{1}(a+c)$. Therefore $J_{13}^{2} \neq 0$, and $J_{23}^{2}=\left(J_{13}^{2}\right)^{*} \neq 0$. We can find $x \in J_{13}$ and $y \in J_{23}$ such that $x^{n}=a+c$ and $y^{m}=b+c$ for some positive integers $n$ and $m$. Then $a$ and $b$ are connected by an element in $J_{12}$ which contradicts $J_{12}=0$.

Assertion 5. Case (3) is impossible.

Proof. Let $J=J_{11}+J_{22}+J_{33}+J_{44}+J_{12}+J_{13}+J_{14}+J_{23}+J_{24}$ $+J_{34}$ be the Peirce decomposition of $J$ with respect to $a, b, c$ and $d$, where $1=a+b+c+d, a^{*}=b, a+b=e_{1}, c+d=e_{2}, c^{*}=d, J_{i i}$ are periodic fields, $J_{12}=J_{1 / 2}(a) \cap J_{1 / 2}(b)=0$ and $J_{34}=J_{1 / 2}(c) \cap J_{1 / 2}(d)=0$. But $J_{13} \neq 0$. Otherwise $J_{24}=J_{13}^{*}=0$ and $J=\left(J_{11}+J_{14}+J_{44}\right)+\left(J_{22}+J_{23}+J_{33}\right)$ contrary to the simplicity of $J$. Similarly, $J_{23} \neq 0$. Then by the same argument in Assertion 4, $a$ and $b$ are connected in $J_{12}=0$, which cannot happen.

Assertion 6. Case (4) is impossible.

Proof. There exist four orthogonal idempotents $a, b, c, d$ such that $a+$ $b=e_{1}, c+d=e_{2}, J_{12}=J_{1 / 2}(a) \cap J_{1 / 2}(b)=0, J_{i i}(i=1,2,3,4)$ are periodic fields. By assumption,

$$
J_{1 / 2}(a+b) \cap J_{1 / 2}(c+d)=J_{13}+J_{14}+J_{23}+J_{24} \supseteq S_{1 / 2}\left(e_{1}\right) \cap S_{1 / 2}\left(e_{2}\right) \neq 0 .
$$

Hence at least one of the $J_{i j} \neq 0$. Without loss of generality, we let $J_{13} \neq 0$. Then $a$ and $c$ are connected and so $a$ and $d$ are connected. This means $J_{14} \neq 0$. If $J_{23}=J_{24}=0$, then we have

$$
J=J_{22}+\left(J_{11}+J_{33}+J_{44}+J_{13}+J_{14}+J_{34}\right)
$$

contrary to the simplicity of $J$. Thus, either $J_{23}$ or $J_{24}$ is not equal to zero. But this implies $a$ and $b$ are connected which contradicts $J_{12}=0$.

Assertion 7. Case (5) is impossible.

Proof. We can find three orthogonal idempotents $a, b, c$ such that $a=$ $e_{1}, b+c=e_{2}$. Let

$$
0 \neq u_{1 / 2} \in S_{1 / 2}\left(e_{1}\right) \cap S_{1 / 2}\left(e_{2}\right) \subseteq J_{1 / 2}(a) \cap J_{1 / 2}(b+c)=J_{12}+J_{13}
$$




$$
u_{1 / 2}=u_{12}+u_{13} \in J_{12}+J_{13}
$$

where $u_{12} \in J_{12}, u_{13} \in J_{13}$. We have $u_{12}$ and $u_{13}$ both not equal to zero, since $u_{1 / 2}$ connects $e_{1}$ and $e_{2}$. Hence $a, b$ and $c$ are connected idempotents. By Theorem 2.3 and Theorem $2.14, J$ is isomorphic to a $3 \times 3$ simple Jordan matrix algebra over a $j$ simple alternative ring $(D, j)$ with involution $j$ : $x \rightarrow \bar{x}$ where $D$ is one of the following:

(i) a periodic field with involution,

(ii) a direct sum of two periodic fields with exchange involution,

(iii) a $2 \times 2$ matrix algebra over a periodic field with standard involution,

(iv) a Cayley-Dickson algebra with standard involution, and

$$
\left.J \sim\left\{\begin{array}{ccc}
f & \bar{g} \mu & \bar{h} \nu \\
g & p & \bar{q} \mu^{-1} \nu \\
h & q & w
\end{array}\right] \mid \begin{array}{l}
f, g, h, p, q, w \in D ; \\
\bar{f}=f, \bar{p}=p, \bar{q}=q, \bar{u}=u, \bar{v}=v
\end{array}\right\}
$$

such that

$$
u_{12}=\left[\begin{array}{lll}
0 & \mu & 0 \\
1 & 0 & 0 \\
0 & 0 & 0
\end{array}\right] \text { and } u_{13}=\left[\begin{array}{lll}
0 & 0 & \nu \\
0 & 0 & 0 \\
1 & 0 & 0
\end{array}\right]
$$

Hence

$$
u_{1 / 2}^{2}=\left[\begin{array}{lll}
0 & \mu & \nu \\
1 & 0 & 0 \\
1 & 0 & 0
\end{array}\right]^{2}=\left[\begin{array}{ccc}
\mu+\nu & 0 & 0 \\
0 & \mu & \nu \\
0 & \mu & \nu
\end{array}\right]
$$

and $\left[\begin{array}{ll}\mu & \nu \\ \mu & \nu\end{array}\right] \in S_{1}(b+c)$ should be invertible in $S_{1}(b+c)$. But $\left[\begin{array}{cc}\mu & \nu \\ \nu\end{array}\right]$ has no inverse. This proves Assertion 7.

Assertion 8. If $J_{i}(i=1,0)$ are both of the same type (c), then $J$ is a $4 \times 4$ Jordan matrix algebra over a ${ }^{*}$-simple associative ring $D$ which is one of the following:

(1) a periodic field,

(2) a direct sum of two periodic fields,

(3) a $2 \times 2$ matrix algebra over a periodic field with standard involution.

Proof. We know from Lemma 3.12 and Lemma 3.13 that $J_{1}\left(e_{i}\right)$ either contains interchanged orthogonal idempotents $a_{i}, b_{i}$ or $S_{1}\left(e_{i}\right) \cap J_{1}\left(e_{i}\right)=S_{i i}$ can be decomposed into $S_{i i}=S_{i i}(+)+S_{i i}(-)$ where $\left[S_{i i}: S_{i i}(+)\right]=2$. In this second case, let $k_{i}$ be any invertible skew element in $J_{1}\left(e_{i}\right)$ and $s_{i} \in S_{i i}(-)$. 
Then there exists $m, n \in S_{i i}(+)$ such that $\left(m s_{i}+n k_{i}\right)^{2}=s^{2}$ for some $s \in$ $S_{i i}(+)$ by Theorem 2.8. Hence $J_{1}\left(e_{i}\right)$ contains orthogonal idempotents $a_{i}=$ $2^{-1}-2^{-1}\left(s^{-1} m s_{i}+s^{-1} n k_{i}\right)$ and $b_{i}=2^{-1}+2^{-1}\left(s^{-1} m s_{i}+s^{-1} n k_{i}\right)$. These two orthogonal idempotents $a_{i}$ and $b_{i}(i=1$, or 2$)$ might not satisfy $a_{i}^{*}=$ $b_{i}$. But if we define $\left(s_{+}+s_{-}+k\right)^{* \prime}=s_{+}-s_{-}-k$, then ${ }^{* \prime}$ is a new involution on $J_{i i}$ by Lemma $3.13(1)$ and also $a_{i}^{* \prime}=b_{i}$. Therefore $J_{1}\left(a_{i}\right)$ and $J_{1}\left(b_{i}\right)$ are periodic fields. It is clear that $a_{1}, a_{2}, b_{1}, b_{2}$ are connected. Then applying Theorem 2.3 and Theorem 2.14 , we get the result.

Assertion 9. The associative ring $D$ in the above assertion is a periodic field with identity involution or both $J_{i i}$ contain interchanged orthogonal idempotents.

Proof. We can find four orthogonal idempotents $a, b, c, d$ such that $a+b=e_{1}, c+d=e_{2}$ and

$$
\begin{gathered}
\left.J \sim\left\{\left[\begin{array}{cccc}
m & \bar{n} \mu & \bar{p} \nu & \bar{q} z \\
n & r & \mu^{-1} \bar{s} \nu & \mu^{-1} \overline{t z} \\
p & s & w & \nu^{-1} \bar{x} z \\
q & t & x & y
\end{array}\right]\right) m, n, p, q, r, s, t, w, x, y \in D\right\}, \\
\bar{m}=m, \bar{r}=r, \bar{w}=w, \bar{y}=y, \bar{\mu}=\mu, \bar{\nu}=\nu, \bar{z}=z .
\end{gathered}
$$

If both $J_{i i}(i=1$, or 2$)$ contain exchanged orthogonal idempotents then we get what we want; if not, we might assume $S_{11}=S_{1}\left(e_{1}\right) \cap J_{1}\left(e_{1}\right)=$ $S_{11}(+)+S_{11}(-)$ where $\left[S_{11}: S_{11}(+)\right]=2$. Let $s=\left[\begin{array}{cc}m & \bar{n} \mu \\ p\end{array}\right] \in S_{11}(-)$. Then

$$
s^{2}=\left[\begin{array}{cc}
m^{2}+n \bar{n} \mu & (m \bar{n}+\bar{n} p) \mu \\
m n+n p & n \bar{n} \mu+p^{2}
\end{array}\right] \in S_{11}(+)
$$

which is the center of $J_{1}\left(e_{1}\right)$ and so $(m+p)(m-p)=0,(m+p) n=0$. If $m+p \neq 0$, we have $m=p, n=0$ and $s=\left[\begin{array}{cc}m & 0 \\ 0 & m\end{array}\right] \in S_{11}(+) \cap S_{11}(-)=0$ which contradicts our assumption that $s \neq 0$. So $m=-p$ and $s=$ $\left(\begin{array}{cc}m & \bar{n} \mu \\ n & -m\end{array}\right) \in S_{11}(-)$. We now claim $\left(\begin{array}{cc}0 & \bar{n} \mu \\ n & 0\end{array}\right)^{2} \neq 0$. If zero, then

$$
\left[\begin{array}{cc}
1 & 2^{-1} m^{-1} \bar{n} \mu \\
2^{-1} m^{-1} n & 0
\end{array}\right]=2^{-1} m^{-1}\left\{\left[\begin{array}{cc}
m & \bar{n} \mu \\
n & -m
\end{array}\right]+\left[\begin{array}{cc}
m & 0 \\
0 & m
\end{array}\right]\right\} \in S_{11}
$$

and 


$$
\left[\begin{array}{cc}
1 & 2^{-1} m^{-1} \bar{n} \mu \\
(2 m)^{-1} n & 0
\end{array}\right]^{2}=\left[\begin{array}{cc}
1 & 2^{-1} m^{-1} \bar{n} \mu \\
2^{-1} m^{-1} n & 0
\end{array}\right]
$$

which contradicts the fact that every element in $S_{11}$ is invertible in $J_{11}$. Thus $w=\left[\begin{array}{cc}0 & \bar{n} \mu \\ n & 0\end{array}\right] \neq 0$ connects $a$ and $b$. By Jacobson's coordinatization Theorem 2.14 , there exists an isomorphism $f$ such that

$$
f(a)=1 / 2[11], \quad f(c)=1 / 2[33], \quad f(d)=1 / 2[44]
$$

and

$$
f(w)=\left[\begin{array}{llll}
0 & \mu & 0 & 0 \\
1 & 0 & 0 & 0 \\
0 & 0 & 0 & 0 \\
0 & 0 & 0 & 0
\end{array}\right] .
$$

Hence we can assume $n=1$ and $s=\left(\begin{array}{cc}m & \mu \\ 1 & -m\end{array}\right) \in S_{11}(-)$. But

$$
\left[\begin{array}{cc}
m & \mu \\
1 & -m
\end{array}\right] \cdot\left[\begin{array}{cc}
1 & -m \\
-\mu^{-1} m & -1
\end{array}\right]=0, \quad\left[\begin{array}{cc}
m & \mu \\
1 & -m
\end{array}\right] \cdot\left[\begin{array}{cc}
0 & -k \mu \\
k & 0
\end{array}\right]=0
$$

where $k$ is an invertible skew element in $D$. Hence

$$
k_{1}=\left[\begin{array}{cc}
1 & -m \\
-\mu^{-1} m & -1
\end{array}\right] \text { and } k_{2}=\left[\begin{array}{cc}
0 & -k \mu \\
k & 0
\end{array}\right]
$$

are skew elements in $J_{1}\left(e_{1}\right)$. Since $k_{1}^{2} \neq 0$ and $k_{2}^{2} \neq 0, k_{1} \cdot k_{2}=0$, there exist $h_{1}, h_{2} \in S_{11}(+)$ such that $\left(h_{1} \cdot k_{1}+h_{2} \cdot k_{2}\right)^{2}=h_{3}^{2}$ for some $h_{3} \in$ $S_{11}(+)$. Hence we can find a skew element $k_{3}=h_{3}^{-1} \cdot h_{1} \cdot k_{1}+h_{3}^{-1} \cdot h_{2} \cdot$ $k_{2}$ such that $k_{3}^{2}=1$. This implies that $2^{-1}\left(1-k_{3}\right)$ and $2^{-1}\left(1+k_{3}\right)$ are interchanged orthogonal idempotents. This contradiction arises when we assume $D$ contains an invertible skew element. Hence $D$ must be a periodic field with identity involution if one of the $J_{1}\left(e_{i}\right)(i=1,2)$ contains no interchanged orthogonal idempotents.

Assertion 10. If both $J_{1}\left(e_{1}\right)$ and $J_{1}\left(e_{2}\right)$ are of the same type (c), then it is impossible that both $J_{1}\left(e_{1}\right)$ and $J_{1}\left(e_{2}\right)$ contain exchanged orthogonal idempotents.

Proof. Let $1=e_{1}+e_{2}$ and $a, b, c, d$ be orthogonal idempotents such that $a+b=e_{1}, c+d=e_{2}, a^{*}=b, c^{*}=d$. Then 


$$
\begin{array}{r}
\left.J \sim\left\{\begin{array}{cccc}
m & \bar{n} \mu & \bar{p} \nu & \bar{q} z \\
n & r & \mu^{-1} s \nu & \mu^{-1} \overline{t z} \\
p & s & w & \nu^{-1} \bar{x} z \\
q & t & x & y
\end{array}\right] \mid m, n, p, q, r, s, t, w, x, y \in D\right\}, \\
\bar{m}=m, \bar{r}=r, \bar{w}=w, \bar{y}=y, \bar{\mu}=\mu, \bar{\nu}=\nu, \bar{z}=z
\end{array}
$$

where $D$ is an associative ring listed in Assertion 8. Since $\left[J_{1}(a+c)\right]^{*}=$ $J_{1}(b+d), D$ is a periodic field with identity involution. Let

$$
\begin{aligned}
& {\left[\begin{array}{llll}
0 & 0 & \nu & 0 \\
0 & 0 & 0 & 0 \\
1 & 0 & 0 & 0 \\
0 & 0 & 0 & 0
\end{array}\right]^{*}=\left[\begin{array}{cccc}
0 & 0 & 0 & 0 \\
0 & 0 & 0 & \mu^{-1} t z \\
0 & 0 & 0 & 0 \\
0 & t & 0 & 0
\end{array}\right]} \\
& {\left[\begin{array}{llll}
0 & 0 & 0 & z \\
0 & 0 & 0 & 0 \\
0 & 0 & 0 & 0 \\
1 & 0 & 0 & 0
\end{array}\right]^{*}=\left[\begin{array}{cccc}
0 & 0 & 0 & 0 \\
0 & 0 & s u^{-1} v & 0 \\
0 & s & 0 & 0 \\
0 & 0 & 0 & 0
\end{array}\right] .}
\end{aligned}
$$

Then

$$
u_{1}=\left[\begin{array}{cccc}
0 & 0 & v & 0 \\
0 & 0 & 0 & u^{-1} t z \\
1 & 0 & 0 & 0 \\
0 & t & 0 & 0
\end{array}\right],\left[\begin{array}{cccc}
0 & 0 & 0 & z \\
0 & 0 & s u^{-1} v & 0 \\
0 & s & 0 & 0 \\
1 & 0 & 0 & 0
\end{array}\right]=u_{2}
$$

are two symmetric elements in $S_{1 / 2}\left(e_{1}\right) \cap S_{1 / 2}\left(e_{2}\right)$. Since $S$ is a simple periodic Jordan ring by our assumption and $u_{1}, u_{2} \in S_{1 / 2}\left(e_{1}\right) \cap S_{1 / 2}\left(e_{2}\right), u_{1} \cdot u_{2} \neq 0$ by Theorem 2.10. By multiplication,

$$
u_{1} \cdot u_{2}=\frac{1}{2}\left[\begin{array}{cccc}
0 & v s+t z & 0 & 0 \\
u^{-1}(t z+s v) & 0 & 0 & 0 \\
0 & 0 & 0 & z\left(1+s u^{-1} t\right) \\
0 & 0 & \left(t s \mu^{-1}+1\right) \nu & 0
\end{array}\right] \neq 0
$$

is an element of $J_{12}+J_{34}$. Since 


$$
u_{1}^{2}=\left[\begin{array}{cccc}
\nu & 0 & 0 & 0 \\
0 & t^{2} u^{-1} z & 0 & 0 \\
0 & 0 & \nu & 0 \\
0 & 0 & 0 & t^{2} u^{-1} z
\end{array}\right] \in S, \quad \nu=t^{2} \mu^{-1} z
$$

Thus $v s+t z=0$ implies $t s u^{-1}+1=t(-t z / v) \mu^{-1}+1=0$. Also $t s u^{-1}+$ $1=0$ implies $v s+t z=v(-\mu / t)+t z=0$. Therefore $S \cap J_{12}$ and $S \cap J_{34}$ are both nonzero. But $J_{12}$ and $J_{34}$ are both one-dimensional over the center and $J_{12}^{*} \subseteq J_{12}, J_{34}^{*} \subseteq J_{34}$ and also the center $Z$ of $J$ is contained in $S$ by our assumption, so $J_{12}$ and $J_{34}$ consist only of symmetric elements. Then

$$
\begin{aligned}
S & =Z+Z \cdot\left(e_{1}-e_{2}\right)+Z \cdot\left[\begin{array}{cccc}
0 & \jmath & \nu & 0 \\
0 & 0 & 0 & u^{-1} t z \\
1 & 0 & 0 & 0 \\
0 & t & 0 & 0
\end{array}\right] \\
& +Z \cdot\left[\begin{array}{cccc}
0 & 0 & 0 & z \\
0 & 0 & s \mu^{-1} \nu & 0 \\
0 & s & 0 & 0 \\
1 & 0 & 0 & 0
\end{array}\right]+Z \cdot\left[\begin{array}{cccc}
0 & \mu & 0 & 0 \\
1 & 0 & 0 & 0 \\
0 & 0 & 0 & 0 \\
0 & 0 & 0 & 0
\end{array}\right] \\
& +Z \cdot\left[\begin{array}{cccc}
0 & 0 & 0 & 0 \\
0 & 0 & 0 & 0 \\
0 & 0 & 0 & \nu^{-1} z \\
0 & 0 & 1 & 0
\end{array}\right]
\end{aligned}
$$

is six-dimensional over $Z$. But by Theorem 2.10, $S$ is 3-dimensional over the center $F$ of $S$. Thus $F$ is two-dimensional over $Z$. Since $u_{1} \in S_{1 / 2}\left(e_{1}\right)$ and $u_{1}^{2} \in Z$, we can find an element $h \in F$ such that $h^{2}=-u^{2}$. Then $\left[h \cdot\left(e_{1}-e_{2}\right)+u_{1}\right]^{2}=h^{2}+u_{1}^{2}=0$ which contradicts the fact that $S$ is periodic. The following two examples show that $J$ can be a $4 \times 4$ Jordan matrix algebra over a periodic field. 


$$
J=\left\{\left[\begin{array}{llll}
m & n & 2 p & q \\
n & r & 2 s & t \\
p & s & w & 2 x \\
q & t & x & y
\end{array}\right] \mid \begin{array}{l}
m, n, p, q, r, s, t, \\
w, x, y \in Z_{3}=\{0,1,2\}
\end{array}\right\}
$$

and

$$
\begin{aligned}
& s_{1}(+)=\left[\begin{array}{llll}
1 & 0 & 0 & 0 \\
0 & 1 & 0 & 0 \\
0 & 0 & 0 & 0 \\
0 & 0 & 0 & 0
\end{array}\right], \quad s_{1}(-)=\left[\begin{array}{cccc}
1 & 1 & 0 & 0 \\
1 & -1 & 0 & 0 \\
0 & 0 & 0 & 0 \\
0 & 0 & 0 & 0
\end{array}\right], \\
& k_{1}=\left[\begin{array}{cccc}
1 & 2 & 0 & 0 \\
2 & -1 & 0 & 0 \\
0 & 0 & 0 & 0 \\
0 & 0 & 0 & 0
\end{array}\right], \quad s_{2}(+)=\left[\begin{array}{llll}
0 & 0 & 0 & 0 \\
0 & 0 & 0 & 0 \\
0 & 0 & 1 & 0 \\
0 & 0 & 0 & 1
\end{array}\right] \text {, } \\
& s_{2}(-)=\left[\begin{array}{llll}
0 & 0 & 0 & 0 \\
0 & 0 & 0 & 0 \\
0 & 0 & 0 & 2 \\
0 & 0 & 1 & 0
\end{array}\right], \quad k_{2}=\left[\begin{array}{rrrr}
0 & 0 & 0 & 0 \\
0 & 0 & 0 & 0 \\
0 & 0 & 1 & 0 \\
0 & 0 & 0 & -1
\end{array}\right] \text {, } \\
& u_{1}=\left[\begin{array}{cccc}
0 & 0 & -2 & 1 \\
0 & 0 & 1 & 0 \\
-1 & 2 & 0 & 0 \\
1 & 0 & 0 & 0
\end{array}\right], \quad u_{2}=\left[\begin{array}{cccc}
0 & 0 & -1 & 1 \\
0 & 0 & 0 & 1 \\
1 & 0 & 0 & 0 \\
1 & 1 & 0 & 0
\end{array}\right] \text {, } \\
& v_{1}=\left[\begin{array}{cccc}
0 & 0 & 0 & 1 \\
0 & 0 & 1 & 2 \\
0 & -1 & 0 & 0 \\
1 & 2 & 0 & 0
\end{array}\right], \quad v_{2}=\left[\begin{array}{llll}
0 & 0 & 2 & 0 \\
0 & 0 & 1 & 1 \\
1 & 2 & 0 & 0 \\
0 & 1 & 0 & 0
\end{array}\right] \text {. }
\end{aligned}
$$

Then the elements listed above form a basis of $J$ over the center $D$. If we define an involution ${ }^{*}$ on $J$ such that $s_{1}(+), s_{1}(-), s_{2}(-), u_{1}, u_{2}$ and the center $D$ are symmetric, $k_{1}, k_{2}, v_{1}$ and $v_{2}$ are skew, the set of symmetric elements forms a simple periodic Jordan algebra of capacity two. We also notice that $J_{1}\left(e_{1}\right)$ has no interchanged orthogonal idempotents and $J_{1}\left(e_{2}\right)$ contains two interchanged orthogonal idempotents in Example (1).

EXAMPLE (2). Let

$$
J=\left\{\left[\begin{array}{llll}
m & n & p & q \\
n & r & s & t \\
p & s & w & x \\
q & t & x & y
\end{array}\right] \begin{array}{l}
m, n, p, q, r, s, t, \\
w, x, y \in Z_{3}=\{0,1,2\}
\end{array}\right\}
$$


and $D$ be the center. Then

$$
\begin{array}{rlrl}
s_{1}(+) & =\left[\begin{array}{cccc}
1 & 0 & 0 & 0 \\
0 & 1 & 0 & 0 \\
0 & 0 & 0 & 0 \\
0 & 0 & 0 & 0
\end{array}\right], & s_{1}(-) & =\left[\begin{array}{cccc}
1 & 1 & 0 & 0 \\
1 & -1 & 0 & 0 \\
0 & 0 & 0 & 0 \\
0 & 0 & 0 & 0
\end{array}\right], \\
k_{1} & =\left[\begin{array}{cccc}
1 & 2 & 0 & 0 \\
2 & -1 & 0 & 0 \\
0 & 0 & 0 & 0 \\
0 & 0 & 0 & 0
\end{array}\right], & s_{2}(+) & =\left[\begin{array}{cccc}
0 & 0 & 0 & 0 \\
0 & 0 & 0 & 0 \\
0 & 0 & 1 & 0 \\
0 & 0 & 0 & 1
\end{array}\right], \\
s_{2}(-) & =\left[\begin{array}{cccc}
0 & 0 & 0 & 0 \\
0 & 0 & 0 & 0 \\
0 & 0 & 1 & 1 \\
0 & 0 & 1 & -1
\end{array}\right], & k_{2}=\left[\begin{array}{cccc}
0 & 0 & 0 & 0 \\
0 & 0 & 0 & 0 \\
0 & 0 & 1 & 2 \\
0 & 0 & 2 & -1
\end{array}\right], \\
u_{1}= & {\left[\begin{array}{cccc}
0 & 0 & 0 & 1 \\
0 & 0 & 2 & 1 \\
0 & 2 & 0 & 0 \\
1 & 1 & 0 & 0
\end{array}\right],} & u_{2}=\left[\begin{array}{cccc}
0 & 0 & 2 & 2 \\
0 & 0 & -2 & 0 \\
2 & -2 & 0 & 0 \\
2 & 0 & 0 & 0
\end{array}\right], \\
v_{1}= & {\left[\begin{array}{cccc}
0 & 0 & 1 & 0 \\
0 & 0 & -2 & 1 \\
1 & -2 & 0 & 0 \\
0 & 1 & 0 & 0
\end{array}\right],} & v_{2}=\left[\begin{array}{cccc}
0 & 0 & 1 & 2 \\
0 & 0 & 0 & 1 \\
1 & 0 & 0 & 0 \\
2 & 1 & 0 & 0
\end{array}\right],
\end{array}
$$

form a basis of $J$ over $D$. We define ${ }^{*}$ on $J$ such that $s_{1}(+), s_{1}(-), s_{2}(+)$, $s_{2}(-), u_{1}, u_{2}$ and $D$ are symmetric elements, $k_{1}, k_{2}, v_{1}$ and $v_{2}$ are skew elements. Then the symmetric part forms a simple periodic Jordan algebra of capacity two. In this example both $J_{1}\left(e_{1}\right)$ and $J_{1}\left(e_{2}\right)$ contain no interchanged orthogonal idempotents.

5. General theorems. In this section, we prove our Main Theorem stated in the abstract.

THEOREM 5.1. Let $S$ be periodic and $N$ be the Jacobson radical of $J$. Then $N=$ the prime radical of $J=$ the intersection of all ${ }^{*}$-prime ideals of $J$.

Proof. Since $N$ contains no idempotent, $N \cap S=0$. But $N^{*} \subseteq$ $N$. This gives us $N^{2}=0$ and $N$ is the maximal nilpotent ideal of $J$. Therefore $N=$ the prime radical of $J$ by Theorem 2.6. Let the prime radical be $R(J)=\bigcap_{\alpha \in I} P_{a}$ where $\left\{P_{a}\right\}_{a \in I}$ is the collection of all prime ideals of $J$ and let $Q(J)$ be the intersection of all * ${ }^{*}$-prime ideals of $J$. Then from Theorem 4.1 any ${ }^{*}$-prime ideal $I$ is either prime or $I=P \cap P^{*}$ where $P$ is prime. Thus any *-prime ideal $I \supseteq R(J)$ and so $Q(J) \supseteq R(J)$. But $R(J)=\bigcap_{a \in I} P_{a}$ $=\bigcap_{a \in I}\left(P_{a} \cap P_{a}^{*}\right)$. Thus $R(J) \supseteq Q(J)$. This implies $R(J)=Q(J)=N$ : 
$N^{2}=0$ and $J / N$ is a subdirect sum of rings of the following types:

(i) a periodic field with involution,

(ii) a direct sum of two simple periodic Jordan algebras interchanged by the involution,

(iii) a $4 \times 4$ Jordan matrix algebra over a periodic field,

(iv) a $3 \times 3$ Jordan matrix algebra over a periodic field,

(v) a Jordan algebra of a nondegenerate symmetric bilinear form over a periodic field.

Proof. We have shown that $N$ is the intersection of all ${ }^{*}$-prime ideals, and so $J / N$ is a subdirect sum of ${ }^{*}$-prime Jordan rings with periodic symmetric elements. If we can show all these ${ }^{*}$-prime Jordan rings are of characteristic not two, then we can apply Theorem 3.14, Theorem 4.8, Theorem 4.9 and Theorem 4.10 to get the above result. The proof of characteristic not two is just the same as the proof in the associative case. See Osborn [3].

\section{REFERENCES}

\section{J. Britten}

[1] Goldie-like conditions on Jordan matrix rings, Ph. D. Thesis, University of Iowa, Iowa City, 1971.

Nathan Jacobson

[1] Structure and representations of Jordan algebras, Amer. Math. Soc. Colloq. Publ., vol. 39, Amer. Math. Soc., Providence, R. I., 1968. MR 40 \#4330.

Kevin McCrimmon

[1] A general theory of Jordan rings, Proc. Nat. Acad. Sci. U.S.A. 56 (1966), 10721079. MR 34 \#2643.

[2] The radical of a Jordan algebra, Proc. Nat. Acad. Sci. U.S.A. 62 (1969), 671-678. MR 42 \#3137.

[3] A characterization of the radical of a Jordan algebra, J. Algebra 18 (1971), 103111. MR 43 \#3316.

S. Montgomery

[1] A generalization of a theorem of Jacobson (preprint).

J. M. Osborn

[1] Jordan algebras of capacity two, Proc. Nat. Acad. Sci. U.S.A. 57 (1967), 582-588. MR 35 \#6727.

[2] Jordan and associative rings with nilpotent and invertible elements, J. Algebra 15 (1970), 301-308. MR 41 \#6925.

[3] Varieties of algebras, Advances in Math., Academic Press, New York, 1972. Chester Tsai

[1] The prime radical in a Jordan ring, Proc. Amer. Math. Soc. 19 (1968), 1171-1175. MR 37 \#6336.

DEPARTMENT OF MATHEMATICS, UNIVERSITY OF IOWA, IOWA CITY, IOWA

Current address: School of Physics and Mathematics, University of Science of Malaysia, Penang, Malaysia 